\title{
ANALYSIS OF AN INCOMPRESSIBLE NAVIER-STOKES-MAXWELL-STEFAN SYSTEM
}

\author{
XIUQING CHEN AND ANSGAR JÜNGEL
}

\begin{abstract}
The incompressible Navier-Stokes equations coupled to the Maxwell-Stefan relations for the molar fluxes are analyzed in bounded domains with no-flux boundary conditions. The system models the dynamics of a multicomponent gaseous mixture under isothermal conditions. The global-in-time existence of bounded weak solutions to the strongly coupled model and their exponential decay to the homogeneous steady state are proved. The mathematical difficulties are due to the singular Maxwell-Stefan diffusion matrix, the cross-diffusion terms, and the Navier-Stokes coupling. The key idea of the proof is the use of a new entropy functional and entropy variables, which allows for a proof of positive lower and upper bounds of the mass densities without the use of a maximum principle.
\end{abstract}

\section{INTRODUCTION}

The dynamics of a multicomponent gaseous mixture can be described by the NavierStokes equations, which represent the balance of mass, momentum, and energy, and the Maxwell-Stefan equations, which model the diffusive transport of the components of the mixture. Applications arise, for instance, from physics (sedimentation, astrophysics), medicine (dialysis, respiratory airways), and chemistry (electrolysis, ion exchange, chemical reactors) 20. The understanding of the analytical structure of coupled Navier-StokesMaxwell-Stefan systems is of great importance for an accurate modeling and efficient numerical simulation of these applications. In this paper, we make a step forward to this understanding by proving the global-in-time existence of weak solutions and their longtime behavior for Navier-Stokes-Maxwell-Stefan systems for incompressible fluids under natural assumptions. This is the first analytical result for the full coupled incompressible model.

More precisely, we consider a multicomponent fluid consisting of $N+1$ components with the mass densities $\rho_{i}$, molar masses $M_{i}$, and velocities $u_{i}$. As in [5], we prescribe a system of partial mass balances together with a common mixture momentum balance, where the

Date: August 16, 2018.

2000 Mathematics Subject Classification. 35K55, 35Q30, 35A01, 35B40, 76D05.

Key words and phrases. Incompressible Navier-Stokes equations, Maxwell-Stefan equations, crossdiffusion, entropy dissipation, entropy variables, global existence of solutions, long-time behavior of solutions.

The first author acknowledges support from the National Science Foundation of China, grant 11101049. The second author was partially supported by the Austrian Science Fund (FWF), grants P22108, P24304, I395, and W1245, and the Austrian-French Project of the Austrian Exchange Service (ÖAD). 
diffusive fluxes are given by the Maxwell-Stefan relations. The partial mass balances for the molar concentrations $c_{i}=\rho_{i} / M_{i}$ read as

$$
\partial_{t} c_{i}+\operatorname{div}\left(j_{i}+c_{i} u\right)=0, \quad i=1, \ldots, N+1,
$$

where the barycentric velocity $u$ and the total mass density $\rho^{*}$ of the fluid are defined by $\rho^{*} u=\sum_{i=1}^{N+1} \rho_{i} u_{i}$ and $\rho^{*}=\sum_{i=1}^{N+1} \rho_{i}$, and the molar mass fluxes $j_{i}$ are given by $j_{i}=$ $c_{i}\left(u_{i}-u\right)$. By definition of $j_{i}$, it holds that $\sum_{i=1}^{N+1} M_{i} j_{i}=0$, and therefore, one of the partial mass balances can be replaced by the continuity equation $\partial_{t} \rho^{*}+\operatorname{div}\left(\rho^{*} u\right)=0$. The mixture momentum balance equations are

$$
\partial_{t}\left(\rho^{*} u\right)+\operatorname{div}\left(\rho^{*} u \otimes u-S\right)+\nabla p=\rho^{*} f
$$

where $p$ is the pressure, the force density equals $\rho^{*} f=\sum_{i=1}^{N+1} \rho_{i} f_{i}$, and the viscous stress tensor is $S=\nu^{*}\left(\nabla u+\nabla u^{\top}\right)$, where $\nu^{*}$ is the viscosity constant. In this paper, we suppose that $f_{i}=f$ and we impose the incompressibility conditions

$$
\rho^{*}=\text { const. }, \quad \operatorname{div} u=0 .
$$

For simplicity, we set $\rho^{*}=1$ and $\nu^{*}=1$.

The above equations are closed by relating the molar mass fluxes $j_{i}$ to the molar concentrations $c_{i}$ by the Maxwell-Stefan equations

$$
-\sum_{k=1}^{N+1} \frac{x_{k} j_{i}-x_{i} j_{k}}{D_{i k}}=c_{i} \nabla \mu_{i}-y_{i} \nabla p-\rho_{i}\left(f_{i}-f\right), \quad i=1, \ldots, N+1,
$$

where $x_{i}=c_{i} / c$ with $c=\sum_{k=1}^{N+1} c_{i}$ are the molar fractions, $y_{i}=\rho_{i} / \rho^{*}=\rho_{i}$ are the mass fractions, $\mu_{i}$ are the molar-based chemical potentials, and $D_{i k}=D_{k i}>0$ for $i \neq k$ are the diffusion coefficients. Our second assumption is that the mixture of gases is ideal such that the chemical potentials can be written as $\mu_{i}=\ln x_{i}+\mu_{0 i}(p)$ with $d \mu_{0 i} / d p=\phi_{i} / c_{i}$, where $\phi_{i}$ is the volume fraction (see [5, Section 1.1]). Since $f_{i}=f$, this implies that

$$
-\sum_{k=1}^{N+1} \frac{x_{k} j_{i}-x_{i} j_{k}}{D_{i k}}=\frac{c_{i}}{x_{i}} \nabla x_{i}+\left(\phi_{i}-y_{i}\right) \nabla p=c \nabla x_{i}+\left(\phi_{i}-y_{i}\right) \nabla p .
$$

We assume further that the volume and mass fractions are comparable such that the contribution $\left(\phi_{i}-y_{i}\right) \nabla p$ can be neglected. This gives the desired closure relations

$$
-\sum_{k=1}^{N+1} \frac{x_{k} j_{i}-x_{i} j_{k}}{D_{i k}}=c \nabla x_{i}, \quad i=1, \ldots, N+1 .
$$

These relations, together with the mass balance equations, can also be derived from a system of kinetic equations with BGK-type collision operator in the Chapman-Enskog expansion [3].

Setting $J_{i}=M_{i} j_{i}$, the incompressible Navier-Stokes-Maxwell-Stefan system analyzed in this paper reads as

$$
\partial_{t} \rho_{i}+\operatorname{div}\left(J_{i}+\rho_{i} u\right)=0, \quad \text { in } \Omega, t>0,
$$




$$
\begin{aligned}
& \partial_{t} u+(u \cdot \nabla) u-\Delta u+\nabla p=f, \quad \operatorname{div} u=0, \\
& \nabla x_{i}=-\sum_{k=1}^{N+1} \frac{\rho_{k} J_{i}-\rho_{i} J_{k}}{c^{2} M_{i} M_{k} D_{i k}}, \quad i=1, \ldots, N+1,
\end{aligned}
$$

where $x_{i}$ and $\rho_{i}$ are related by $x_{i}=\rho_{i} /\left(c M_{i}\right)$ with $c=\sum_{i=1}^{N+1} \rho_{i} / M_{i}$ and $\Omega \subset \mathbb{R}^{d}(d \leq 3)$ is a bounded domain. Note that $\rho^{*}=1$ implies that $\sum_{i=1}^{N+1} \rho_{i}=\sum_{i=1}^{N+1} x_{i}=1$. The initial and boundary conditions are

$$
\rho_{i}(\cdot, 0)=\rho_{i}^{0}, u(\cdot, 0)=u^{0} \quad \text { in } \Omega, \quad \nabla \rho_{i} \cdot \nu=0, u=0 \quad \text { on } \partial \Omega,
$$

where $i=1, \ldots, N+1$ and $\nu$ is the normal exterior unit vector on $\partial \Omega$.

There are several difficulties to overcome in the analysis of the above system.

First, the molar mass fluxes are not explicitly given as a linear combination of the mass density gradients, which makes necessary to invert the flux-gradient relations (3). However, as the Maxwell-Stefan equations are linearly dependent, we need to invert on a subspace. In the engineering literature, this inversion is usually done in an approximate way [2]. Giovangigli [9] suggested an iterative procedure using the Perron-Frobenius theory. A general inversion result was proved by Bothe [4], again based on the Perron-Frobenius theory.

Second, equations (11)-(3) are strongly coupled through the Maxwell-Stefan relations (3) and the velocity $u$, computed from the Navier-Stokes equations. Because of the crossdiffusion coupling in (11) and (3), standard tools like maximum principles and regularity theory are not available. In particular, it is not clear how to prove positive lower and upper bounds for the mass densities $\rho_{i}$ and even the local existence of solutions is not trivial.

Third, we need to find suitable a priori estimates for the coupled system. The energy method provides gradient estimates for the velocity, but it is less clear how to derive estimates for the mass densities. Moreover, the velocity does not need to be bounded such that the term $\operatorname{div}\left(\rho_{i} u\right)$ in (11) needs to be treated carefully.

In view of these difficulties, it is not surprising that there exist only partial results on such systems in the literature. First results were concerned with the Maxwell-Stefan equations (11) and (3) with vanishing velocity $u=0$ and equal molar masses $M=M_{i}$. Griepentrog [12] and later Bothe [4] derived a local existence theory; Giovangigli [10, Theorem 9.4.1] proved the global existence of solutions with initial data sufficiently close to the equilibrium state; Boudin, Grec, and Salvarani [6] investigated a particular two-component model; and Jüngel and Stelzer [14] presented general global existence results. The Maxwell-Stefan system with given bounded velovity $u \neq 0$ was analyzed by Mucha, Pokorný, and Zatorska [17. They imposed a special diffusion matrix which avoids the inversion problem.

Other papers were concerned with the full coupled system but in particular situations. For instance, Zatorska [21] proved the existence of weak solutions to the stationary compressible model with three fluid components and special isobaric pressures. She also proved the sequential stability of weak solutions to the two-component system on the threedimensional torus [22]. Mucha, Pokorný, and Zatorska [16] showed a global existence 
result for a regularized compressible system for two components. The Navier-Stokes equations contain artificial higher-order differential operators which regularize the problem. In [15], the global existence for the incompressible Navier-Stokes-Maxwell-Stefan system was announced but not proved. For numerical approximations using a finite-volume method, we refer to [1].

In this paper, we prove a general global existence result for the full coupled system (11)-(4), allowing for different molar masses $M_{i}$. We overcome the above difficulties by combining analytical tools for the incompressible Navier-Stokes equations due to Temam [18; the Perron-Frobenius theory for the matrix inversion problem exploited by Bothe [4]; and the entropy-dissipation method developed for cross-diffusion systems in [7, 14]. We detail our key ideas below.

In order to state our first main result, we introduce the following spaces (see [18, Chapter I]). Let $\Omega \subset \mathbb{R}^{d}$ be a bounded domain with $\partial \Omega \in C^{1,1}$ and let

$$
\begin{aligned}
\mathcal{H} & =\left\{u \in L^{2}\left(\Omega ; \mathbb{R}^{d}\right): \operatorname{div} u=0,\left.u \cdot \nu\right|_{\partial \Omega}=0\right\}, \\
\mathcal{V} & =\left\{u \in H_{0}^{1}\left(\Omega ; \mathbb{R}^{d}\right): \operatorname{div} u=0\right\}, \quad \mathcal{V}_{2}=\mathcal{V} \cap H^{2}\left(\Omega ; \mathbb{R}^{d}\right), \\
\widetilde{H}^{2}\left(\Omega ; \mathbb{R}^{N}\right) & =\left\{q \in H^{2}\left(\Omega ; \mathbb{R}^{N}\right):\left.\nabla q \cdot \nu\right|_{\partial \Omega}=0\right\} .
\end{aligned}
$$

We define similarly the space $\widetilde{H}^{2}(\Omega)$. We recall that functions $u \in L^{2}\left(\Omega ; \mathbb{R}^{d}\right)$ with $\operatorname{div} u \in$ $L^{2}(\Omega)$ satisfy $\left.u \cdot \nu\right|_{\partial \Omega} \in H^{-1 / 2}(\partial \Omega)$ such that the space $\mathcal{H}$ is well defined [18, Theorem I.1.2].

Theorem 1 (Global existence). Let $d=1,2,3, T>0$, and $D_{i j}=D_{j i}>0$ for $i, j=$ $1, \ldots, N+1, i \neq j$. Suppose that $f \in L^{2}\left(0, T ; \mathcal{V}^{\prime}\right), u^{0} \in \mathcal{H}$, and let $\rho_{1}^{0}, \ldots, \rho_{N+1}^{0} \in L^{1}(\Omega)$ be nonnegative functions which satisfy $\sum_{i=1}^{N+1} \rho_{i}^{0}=1$ and $h\left(\rho^{0}\right)<+\infty$, where $\rho^{0}=\left(\rho_{1}^{0}, \ldots, \rho_{N}^{0}\right)$ and $h$ is defined in (7) below. Then there exists a global weak solution $\left(u, \rho_{1}, \ldots, \rho_{N+1}\right)$ to (11) -(41) (in the sense of (27)-(28) below) such that $\rho_{i} \geq 0, \sum_{j=1}^{N+1} \rho_{i}=1$ in $\Omega \times(0, T)$, and

$$
\begin{aligned}
& u \in L^{\infty}(0, T ; \mathcal{H}) \cap L^{2}(0, T ; \mathcal{V}), \quad \partial_{t} u \in L^{2}\left(0, T ; \mathcal{V}_{2}^{\prime}\right) \\
& \rho_{i} \in L^{2}\left(0, T ; H^{1}(\Omega)\right), \quad \partial_{t} \rho_{i} \in L^{2}\left(0, T ; \widetilde{H}^{2}(\Omega)^{\prime}\right), \quad i=1, \ldots, N+1
\end{aligned}
$$

We stress the fact that although the diffusion coefficients $D_{i j}$ are constant, the diffusion matrix of the inverted Maxwell-Stefan system (see (6) below) depends on the mass densities in a nonlinear way. Note that the same existence result holds when we allow for reaction terms in (11) which are locally Lipschitz continuous and quasi-positive; see [4, 14].

The key ideas of the proof are as follows. First, we write (3) more compactly as $\nabla x=$ $A(\rho) J$, where $x=\left(x_{1}, \ldots, x_{N+1}\right), \rho=\left(\rho_{1}, \ldots, \rho_{N+1}\right), J=\left(J_{1}, \ldots, J_{N+1}\right)$, and $A(\rho)$ is a matrix. Using the Perron-Frobenius theory, Bothe [4] proved that $A(\rho)$ can be inverted on its image. As in [14], it turns out that it is more convenient to work with the system in $N$ components by eliminating the last equation in (11). We set $x^{\prime}=\left(x_{1}, \ldots, x_{N}\right)$ and similarly for the other vectors. Then, inverting $\nabla x^{\prime}=-A_{0}(\rho) J^{\prime}$ (Lemma 3), (1) becomes

$$
\partial_{t} \rho^{\prime}+(u \cdot \nabla) \rho^{\prime}-\operatorname{div}\left(A_{0}(\rho)^{-1} \nabla x^{\prime}\right)=0 .
$$


This equation can be analyzed by exploiting its entropy structure. Indeed, we associate to this system the entropy density (or, more precisely, Gibbs free energy)

$$
h\left(\rho^{\prime}\right)=c \sum_{i=1}^{N+1} x_{i}\left(\ln x_{i}-1\right)+c
$$

where $\rho_{N+1}=1-\sum_{i=1}^{N} \rho_{i}$ is interpreted as a function of the other mass densities. We "symmetrize" (6) by introducing the entropy variables

$$
w_{i}=\frac{\partial h}{\partial \rho_{i}}=\frac{\ln x_{i}}{M_{i}}-\frac{\ln x_{N+1}}{M_{N+1}}, \quad i=1, \ldots, N,
$$

and set $w=\left(w_{1}, \ldots, w_{N}\right)$. The second equality in (8) is shown in Lemma 4 below. Denoting by $D^{2} h\left(\rho^{\prime}\right)$ the Hessian of $h$ with respect to $\rho^{\prime}$, (6) is equivalent to

$$
\partial_{t} \rho^{\prime}+(u \cdot \nabla) \rho^{\prime}-\operatorname{div}(B(w) \nabla w)=0,
$$

where $B(w)=A_{0}^{-1}\left(\rho^{\prime}\right)\left(D^{2} h\right)^{-1}\left(\rho^{\prime}\right)$ is symmetric and positive definite (Lemma 8). This formulation reveals the parabolic structure of the equations. The mass density vector $\rho^{\prime}$ is interpreted as a function of $w$. If all molar masses are equal, $M_{i}=M$, this function can be written as $\rho_{i}(w)=\exp \left(M w_{i}\right)\left(1+\sum_{j=1}^{N} \exp \left(M w_{j}\right)\right)^{-1}$ [14], showing that

$$
0<\rho^{\prime}<1 \text { and } \sum_{i=1}^{N} \rho_{i}<1 .
$$

This formulation is no longer possible if the molar masses are different. In this situation, $\rho^{\prime}$ is implicitly given as a function of $w$; there is no explicit formula anymore. However, we are able to show that the mapping $\rho^{\prime} \mapsto w$, defined by (8) and $x_{i}=\rho_{i} /\left(c M_{i}\right)$, can be inverted and that (10) still holds (Corollary 7).

The entropy $H\left(\rho^{\prime}\right)=\int_{\Omega} h\left(\rho^{\prime}\right) d z$ provides suitable a priori estimates. Indeed, using $w$ as a test function in (9), a computation (see Lemma 11 and the proof of Theorem 11) shows the entropy-dissipation inequality

$$
\frac{d H}{d t}=-\int_{\Omega} \nabla w: B(w) \nabla w d z \leq-C_{B} \sum_{i=1}^{N+1} \int_{\Omega}\left|\nabla \sqrt{x_{i}}\right|^{2} d z,
$$

where the constant $C_{B}>0$ only depends on the diffusion coefficients $D_{i j}$ and the molar masses $M_{i}$ and the double point ":" signifies summation over both matrix indices. The key point is that the integral $\int_{\Omega}\left((u \cdot \nabla) \rho^{\prime}\right) \cdot w d z$ in (9) vanishes (Lemma 10). This yields $H^{1}$ estimates for $\sqrt{x_{i}}$ from which we conclude $H^{1}$ bounds for $\rho_{i}$ (Lemma 14). We note that a diffusion inequality which directly implies the above entropy-dissipation inequality was first established in [11, Section 4].

The proof of Theorem 1 is based on a semi-discretization in time of both the NavierStokes equations (2) and Maxwell-Stefan equations (9) with time step $\tau>0$, together with a regularization using the operator $\varepsilon\left(\Delta^{2} w+w\right)$ in (9), which guarantees the coercivity in $w$. The existence of a solution to the approximate problem is shown by means of the Leray-Schauder fixed-point theorem. The discrete analogon of the entropy-dissipation 
inequality (11) provides bounds uniform in the approximation parameters $\tau$ and $\varepsilon$. By weak compactness and the Aubin lemma, this allows us to perform the $\operatorname{limit}(\tau, \varepsilon) \rightarrow 0$.

System (11)-(3) admits the homogeneous steady state $\bar{\rho}_{i}^{0}=\operatorname{meas}(\Omega)^{-1}\left\|\rho_{i}^{0}\right\|_{L^{1}(\Omega)}$ or $\bar{x}_{i}^{0}=$ $\bar{\rho}_{i}^{0} /\left(\bar{c}^{0} M_{i}\right)$, where $\bar{c}^{0}=\sum_{i=1}^{N+1} \bar{\rho}_{i}^{0} / M_{i}$. We prove that the solution to (1)-(3), constructed in Theorem 1, converges exponentially fast to this stationary state. For this, we introduce the relative entropy

$$
H^{*}(\rho)=\sum_{i=1}^{N+1} \int_{\Omega} c x_{i} \ln \frac{x_{i}}{\bar{x}_{i}^{0}} d z .
$$

Theorem 2 (Exponential decay). Let the assumptions of Theorem 1 hold and let $f=0$. We assume that there exists $0<\eta<1$ such that $\rho_{i}^{0} \geq \eta$ for $i=1, \ldots, N+1$. Let $(u, \rho)$ be the weak solution constructed in Theorem 1. Then there exist constants $C>0$, only depending on $\rho_{i}^{0}$ and $M_{i}$, and $\lambda>0$, only depending on $\Omega$ and $M_{i}$, such that for all $t>0$ and $i=1, \ldots, N+1$,

$$
\left\|x_{i}(\cdot, t)-\bar{x}_{i}^{0}\right\|_{L^{1}(\Omega)} \leq C e^{-\lambda t} \sqrt{H^{*}\left(\rho^{0}\right)}
$$

where $x_{i}=\rho_{i} /\left(c M_{i}\right)$ with $c=\sum_{i=1}^{N+1} \rho_{i} / M_{i}$ and $\bar{x}_{i}^{0}=\rho_{i}^{0} /\left(\bar{c}^{0} M_{i}\right)$ with $\bar{c}^{0}=\sum_{i=1}^{N+1} \bar{\rho}_{i}^{0} / M_{i}$.

The proof is based on the entropy-dissipation inequality (11) by relating the entropy dissipation with the entropy via the logarithmic Sobolev inequality [13, Remark 3.7]. Similarly as in [14], the difficulty of the proof is that the approximate solution does not conserve the $L^{1}$ norm because of the presence of the regularizing $\varepsilon$-terms. The estimations of these terms make the proof rather technical.

Compared to our previous work [14], the main novelties in this paper are the coupling to the Navier-Stokes equations and the molar masses $M_{i}$ which are not equal. Because of the different molar masses, we need to distinguish between the mass densities $\rho_{i}$ and the molar fractions $x_{i}$, which makes necessary to derive some additional estimates. In particular, the proof of the positive definiteness of the Hessian of $h$, which implies the positive definiteness of $B(w)$, is rather involved (see Lemma 8).

The paper is organized as follows. In Section 2, we prove some auxiliary results needed for the main proofs. In particular, we show properties of the relations between $w, \rho$, and $x$ and of the matrices $D^{2} h$ and $B(w)$. The proofs of Theorems 1 and 2 are presented in Sections 3 and 4, respectively.

\section{Preparations}

In this section, we show some auxiliary results which are used in the proofs of the main theorems.

2.1. Equivalent formulation of (11) and (3). We recall the notation $\rho=\left(\rho^{\prime}, \rho_{N+1}\right)$, $\rho^{\prime}=\left(\rho_{1}, \ldots, \rho_{N}\right)$ and similarly for $x$ and $J$, defined by $x_{i}=\rho_{i} /\left(c M_{i}\right)$ and $J_{i}=M_{i} j_{i}$ $(i=1, \ldots, N+1)$. The matrix $\nabla \rho$ consists of the elements $\partial \rho_{i} / \partial z_{j}(1 \leq i \leq N+1$, $1 \leq j \leq d)$, and we define similarly $\nabla x$ and $\nabla w$. Then we can formulate (11) and (3) more 
compactly as

$$
\partial_{t} \rho+(u \cdot \nabla) \rho+\operatorname{div} J=0, \quad \nabla x=A J,
$$

where the $(N+1) \times(N+1)$ matrix $A=A(\rho)=\left(A_{i j}\right)$ is defined by

$$
\begin{aligned}
& A_{i j}=d_{i j} \rho_{i} \quad \text { if } i \neq j, i, j=1, \ldots, N+1, \\
& A_{i j}=-\sum_{k=1, k \neq i}^{N+1} d_{i k} \rho_{k} \quad \text { if } i=j=1, \ldots, N+1,
\end{aligned}
$$

and $d_{i j}=1 /\left(c^{2} M_{i} M_{j} D_{i j}\right)$. It is shown in [14, Section 2] that the system of $N+1$ equations $\nabla x=A J$ can be reduced to the first $N$ components, leading to

$$
\partial_{t} \rho^{\prime}+(u \cdot \nabla) \rho^{\prime}+\operatorname{div} J^{\prime}=0, \quad \nabla x^{\prime}=A_{0} J^{\prime},
$$

where the $N \times N$ matrix $A_{0}=A_{0}\left(\rho^{\prime}\right)=\left(A_{i j}^{0}\right)$ is given by

$$
\begin{aligned}
& A_{i j}^{0}=-\left(d_{i j}-d_{i, N+1}\right) \rho_{i} \quad \text { if } i \neq j, i, j=1, \ldots, N, \\
& A_{i j}^{0}=\sum_{k=1, k \neq i}^{N}\left(d_{i k}-d_{i, N+1}\right) \rho_{k}+d_{i, N+1} \quad \text { if } i=j=1, \ldots, N .
\end{aligned}
$$

Lemma 3. The matrix $A_{0}$ is invertible and the elements of its inverse $A_{0}^{-1}$ are uniformly bounded in $\rho_{1}, \ldots, \rho_{N} \in[0,1]$.

Proof. The definition $c=\sum_{i=1}^{N+1} \rho_{i} / M_{i}$ and the property $0 \leq \rho_{i} \leq 1$ imply that

$$
\left(\max _{1 \leq i \leq N+1} M_{i}\right)^{-1} \leq c \leq\left(\min _{1 \leq i \leq N+1} M_{i}\right)^{-1}
$$

Hence, the coefficients $d_{i j}=1 /\left(c^{2} M_{i} M_{j} D_{i j}\right)$ are bounded uniformly in $\rho_{k} \in[0,1]$. Therefore, the proof of Lemma 2.3 in [14] applies, proving the result.

2.2. Entropy variables. We recall the relations $x_{i}=\rho_{i} /\left(c M_{i}\right), c=\sum_{i=1}^{N+1} \rho_{i} / M_{i}$, and $\sum_{i=1}^{N+1} \rho_{i}=1$. Since

$$
x_{N+1}=\frac{\rho_{N+1}}{c M_{N+1}}=\frac{1}{c M_{N+1}}\left(1-\sum_{i=1}^{N} \rho_{i}\right), \quad c=\sum_{i=1}^{N} \frac{\rho_{i}}{M_{i}}+\frac{1}{M_{N+1}}\left(1-\sum_{i=1}^{N} \rho_{i}\right),
$$

we may interpret the entropy density (7) as a function of $\rho^{\prime}=\left(\rho_{1}, \ldots, \rho_{N}\right)$, which gives

$$
\begin{aligned}
h\left(\rho^{\prime}\right) & =c \sum_{i=1}^{N} x_{i}\left(\ln x_{i}-1\right)+c x_{N+1}\left(\ln x_{N+1}-1\right)+c \\
& =\sum_{i=1}^{N} \frac{\rho_{i}}{M_{i}}\left(\ln \frac{\rho_{i}}{M_{i}}-1\right)+\frac{\rho_{N+1}}{M_{N+1}}\left(\ln \frac{\rho_{N+1}}{M_{N+1}}-1\right)-c(\ln c-1),
\end{aligned}
$$

First, we prove that the entropy variables can be written as in (8). 
Lemma 4. The entropy variables $w_{i}=\partial h\left(\rho^{\prime}\right) / \partial \rho_{i}$ are given by

$$
w_{i}=\frac{\ln x_{i}}{M_{i}}-\frac{\ln x_{N+1}}{M_{N+1}}, \quad i=1, \ldots, N .
$$

Proof. The proof is just a computation. Indeed, we infer from

$$
\frac{\partial c}{\partial \rho_{i}}=\frac{\partial}{\partial \rho_{i}}\left(\sum_{k=1}^{N} \frac{\rho_{k}}{M_{k}}+\frac{1}{M_{N+1}}\left(1-\sum_{k=1}^{N} \rho_{k}\right)\right)=\frac{1}{M_{i}}-\frac{1}{M_{N+1}}
$$

for $i=1, \ldots, N$ that

$$
\begin{aligned}
\frac{\partial h}{\partial \rho_{i}}\left(\rho^{\prime}\right) & =\frac{1}{M_{i}} \ln \frac{\rho_{i}}{M_{i}}-\frac{1}{M_{N+1}} \ln \frac{\rho_{N+1}}{M_{N+1}}-\left(\frac{1}{M_{i}}-\frac{1}{M_{N+1}}\right) \ln c \\
& =\frac{1}{M_{i}} \ln \frac{\rho_{i}}{c M_{i}}-\frac{1}{M_{N+1}} \ln \frac{\rho_{N+1}}{c M_{N+1}},
\end{aligned}
$$

and since $\rho_{i} /\left(c M_{i}\right)=x_{i}$, the conclusion follows.

We claim that we can invert the mapping $x^{\prime} \mapsto w$, defined by (19).

Lemma 5. Let $w=\left(w_{1}, \ldots, w_{N}\right) \in \mathbb{R}^{N}$ be given. Then there exists a unique $\left(x_{1}, \ldots, x_{N}\right) \in$ $(0,1)^{N}$ satisfying $\sum_{i=1}^{N} x_{i}<1$ such that (19) holds with $x_{N+1}=1-\sum_{i=1}^{N} x_{i}$. In particular, the mapping $\mathbb{R}^{N} \rightarrow(0,1)^{N}, x^{\prime}(w)=\left(x_{1}, \ldots, x_{N}\right)$, is bounded.

Proof. Introduce the function $f(s)=\sum_{i=1}^{N}(1-s)^{M_{i} / M_{N+1}} \exp \left(M_{i} w_{i}\right)$ for $s \in[0,1]$. Then $f$ is strictly decreasing in $[0,1]$ and $0=f(1)<f(s)<f(0)=\sum_{i=1}^{N} \exp \left(M_{i} w_{i}\right)$ for $s \in(0,1)$. By continuity, there exists a unique fixed point $s_{0} \in(0,1), f\left(s_{0}\right)=s_{0}$. Defining $x_{i}=\left(1-s_{0}\right)^{M_{i} / M_{N+1}} \exp \left(M_{i} w_{i}\right)$ for $i=1, \ldots, N$, we infer that $x_{i}>0$ and $\sum_{i=1}^{N} x_{i}=$ $f\left(s_{0}\right)=s_{0}<1$. Hence, in view of $x_{N+1}=1-s_{0}$, (19) holds.

Given $\rho$, we can define $x_{i}=\rho_{i} /\left(c M_{i}\right)$, where $c=\sum_{i=1}^{N+1} \rho_{i} / M_{i}$. The following lemma ensures that this mapping is invertible.

Lemma 6. Let $x^{\prime}=\left(x_{1}, \ldots, x_{N}\right) \in(0,1)^{N}$ and $x_{N+1}=1-\sum_{i=1}^{N} x_{i}>0$ be given and define for $i=1, \ldots, N+1$,

$$
\rho_{i}\left(x^{\prime}\right)=\rho_{i}=c M_{i} x_{i}, \quad \text { where } c=\frac{1}{\sum_{k=1}^{N+1} M_{k} x_{k}} .
$$

Then $\left(\rho_{1}, \ldots, \rho_{N}\right) \in(0,1)^{N}$ is the unique vector satisfying $\rho_{N+1}=1-\sum_{i=1}^{N} \rho_{i}>0$, $x_{i}=\rho_{i} /\left(c M_{i}\right)$ for $i=1, \ldots, N+1$, and $c=\sum_{k=1}^{N+1} \rho_{k} / M_{k}$.

The proof follows immediately from $\sum_{k=1}^{N+1} \rho_{k} / M_{k}=\sum_{k=1}^{N+1} c x_{k}=c$, and the fact that $\rho_{i} / M_{i} x_{i}=\sum_{k=1}^{N+1} \rho_{k} / M_{k}$ for $i=1,2, \ldots, N$ have unique solutions $\rho_{i}=M_{i} x_{i} / \sum_{k=1}^{N+1} M_{k} x_{k}$ for $i=1,2, \ldots, N$ by applying Cramer's rule.

Combining Lemmas 5 and 6 , we infer the following result. 
Corollary 7. Let $w=\left(w_{1}, \ldots, w_{N}\right) \in \mathbb{R}^{N}$ be given. Then there exists a unique vector $\left(\rho_{1}, \ldots, \rho_{N}\right) \in(0,1)^{N}$ satisfying $\sum_{i=1}^{N} \rho_{i}<1$ such that (19) holds for $\rho_{N+1}=1-\sum_{i=1}^{N} \rho_{i}$ and $x_{i}=\rho_{i} /\left(c M_{i}\right)$ with $c=\sum_{i=1}^{N+1} \rho_{i} / M_{i}$. Moreover, the mapping $\mathbb{R}^{N} \rightarrow(0,1)^{N}, \rho^{\prime}(w)=$ $\left(\rho_{1}, \ldots, \rho_{N}\right)$, is bounded.

2.3. Hessian of the entropy density. We prove some properties of the Hessian $\left(H_{i j}\right)=$ $\left(\partial^{2} h\left(\rho^{\prime}\right) / \partial \rho_{i} \partial \rho_{j}\right)_{1 \leq i, j \leq N}=\left(\partial w_{i} / \partial \rho_{j}\right)_{1 \leq i, j \leq N}$ and the matrix $\left(G_{i j}\right)=\left(\partial w_{i} / \partial x_{j}\right)_{1 \leq i, j \leq N}$. Differentiating (18) gives

$$
H_{i j}=\frac{\delta_{i j}}{M_{i} \rho_{i}}+\frac{1}{M_{N+1} \rho_{N+1}}-\frac{1}{c}\left(\frac{1}{M_{i}}-\frac{1}{M_{N+1}}\right)\left(\frac{1}{M_{j}}-\frac{1}{M_{N+1}}\right), \quad i, j=1, \ldots, N,
$$

where $\delta_{i j}$ denotes the Kronecker delta.

Lemma 8. The matrix $\left(H_{i j}\right)$ is symmetric and positive definite for all $\rho_{1}, \ldots, \rho_{N}>0$ satisfying $\sum_{i=1}^{N} \rho_{i}<1$.

Proof. We claim that the principal minors $\operatorname{det} H_{k}$ of $\left(H_{i j}\right)$ satisfy

$$
\operatorname{det} H_{k}>\frac{2}{c M_{N+1} \prod_{\ell=1}^{k} M_{\ell}}\left(\sum_{i, j=1, i<j}^{k} \frac{1}{\rho_{N+1} \prod_{\ell=1, \ell \neq i, j}^{k} \rho_{\ell}}+\sum_{j=1}^{k} \frac{1}{\prod_{\ell=1, \ell \neq j}^{k} \rho_{\ell}}\right)>0
$$

for $k=1, \ldots, N$. Then the positive definiteness of $\left(H_{i j}\right)$ follows from Sylvester's criterion. It remains to prove (20). Since each column of $H_{k}$ can be written for $j=1,2, \ldots, k$, as the difference

$$
\left(\begin{array}{c}
\delta_{1 j}\left(M_{1} \rho_{1}\right)^{-1}+\left(M_{N+1} \rho_{N+1}\right)^{-1} \\
\vdots \\
\delta_{k j}\left(M_{k} \rho_{k}\right)^{-1}+\left(M_{N+1} \rho_{N+1}\right)^{-1}
\end{array}\right)-\frac{1}{c}\left(\frac{1}{M_{j}}-\frac{1}{M_{N+1}}\right)\left(\begin{array}{c}
M_{1}^{-1}-M_{N+1}^{-1} \\
\vdots \\
M_{k}^{-1}-M_{N+1}^{-1}
\end{array}\right),
$$

a calculation shows that

$$
\begin{aligned}
\operatorname{det} H_{k}= & \frac{1}{\prod_{\ell=1}^{k} M_{\ell} \rho_{\ell}}\left(\sum_{j=1}^{k} \frac{M_{j} \rho_{j}}{M_{N+1} \rho_{N+1}}+1\right) \\
& -\frac{1}{c} \sum_{j=1}^{k}\left(\frac{1}{M_{j}}-\frac{1}{M_{N+1}}\right) \frac{1}{\prod_{\ell=1, \ell \neq j}^{k} M_{\ell} \rho_{\ell}} \\
& \times\left(\sum_{i=1, i \neq j}^{k} \frac{M_{i} \rho_{i}}{M_{N+1} \rho_{N+1}}\left(\frac{1}{M_{j}}-\frac{1}{M_{i}}\right)+\left(\frac{1}{M_{j}}-\frac{1}{M_{N+1}}\right)\right) .
\end{aligned}
$$

Multiplying this expression by $c$ and rearranging the terms, we find that

$$
c \operatorname{det} H_{k}=\left(\sum_{j=1}^{k} \frac{c}{M_{N+1} \rho_{N+1} \prod_{\ell=1, \ell \neq j}^{k} M_{\ell} \rho_{\ell}}+\frac{c}{\prod_{\ell=1}^{k} M_{\ell} \rho_{\ell}}\right)
$$




$$
\begin{aligned}
& -\sum_{j=1}^{k}\left(\frac{1}{M_{j}}-\frac{1}{M_{N+1}}\right)^{2} \frac{1}{\prod_{\ell=1, \ell \neq j}^{k} M_{\ell} \rho_{\ell}} \\
& -\sum_{j=1}^{k}\left(\frac{1}{M_{j}}-\frac{1}{M_{N+1}}\right) \sum_{i=1, i \neq j}^{k}\left(\frac{1}{M_{j}}-\frac{1}{M_{i}}\right) \frac{M_{i} \rho_{i}}{M_{N+1} \rho_{N+1} \prod_{\ell=1, \ell \neq j}^{k} M_{\ell} \rho_{\ell}} \\
= & I_{1}+I_{2}+I_{3} .
\end{aligned}
$$

Recalling that $c=\sum_{\ell=1}^{N+1} \rho_{\ell} / M_{\ell}$, we can estimate as follows:

$$
\begin{aligned}
I_{1}> & \sum_{j=1}^{k} \frac{\sum_{i=1, i \neq j}^{k} \rho_{i} / M_{i}+\rho_{N+1} / M_{N+1}}{M_{N+1} \rho_{N+1} \prod_{\ell=1, \ell \neq j}^{k} M_{\ell} \rho_{\ell}}+\frac{\sum_{j=1}^{k} \rho_{j} / M_{j}}{\prod_{\ell=1}^{k} M_{\ell} \rho_{\ell}} \\
= & \sum_{j=1}^{k}\left(\sum_{i=1, i \neq j}^{k} \frac{1}{M_{i}^{2} M_{N+1} \rho_{N+1} \prod_{\ell=1, \ell \neq i, j}^{k} M_{\ell} \rho_{\ell}}+\frac{1}{M_{N+1}^{2} \prod_{\ell=1, \ell \neq j}^{k} M_{\ell} \rho_{\ell}}\right) \\
& +\sum_{j=1}^{k} \frac{1}{M_{j}^{2} \prod_{\ell=1, \ell \neq j}^{k} M_{\ell} \rho_{\ell}} \\
= & \sum_{i, j=1, i<j}^{k} \frac{M_{i}^{-2}+M_{j}^{-2}}{M_{N+1} \rho_{N+1} \prod_{\ell=1, \ell \neq i, j}^{k} M_{\ell} \rho_{\ell}}+\sum_{j=1}^{k} \frac{M_{j}^{-2}+M_{N+1}^{-2}}{\prod_{\ell=1, \ell \neq j}^{k} M_{\ell} \rho_{\ell}}
\end{aligned}
$$

Using $\sum_{i, j} b_{i j}\left(a_{j}-a\right)\left(a_{j}-a_{i}\right)=\sum_{i<j} b_{i j}\left(a_{j}-a_{i}\right)^{2}$ for numbers $a, a_{i} \in \mathbb{R}$ and $b_{i j}=b_{j i} \in \mathbb{R}$, the last term $I_{3}$ can be formulated as

$$
I_{3}=-\sum_{j=1}^{k} \sum_{i=1, i \neq j}^{k} \frac{\left(M_{j}^{-1}-M_{N+1}^{-1}\right)\left(M_{j}^{-1}-M_{i}^{-1}\right)}{M_{N+1} \rho_{N+1} \prod_{\ell=1, \ell \neq i, j}^{k} M_{\ell} \rho_{\ell}}=-\sum_{i, j=1, i<j}^{k} \frac{\left(M_{i}^{-1}-M_{j}^{-1}\right)^{2}}{M_{N+1} \rho_{N+1} \prod_{\ell=1, \ell \neq i, j}^{k} M_{\ell} \rho_{\ell}} .
$$

Therefore, we infer that

$$
\begin{aligned}
c \operatorname{det} H_{k} & >\sum_{i, j=1, i<j}^{k} \frac{2 M_{i}^{-1} M_{j}^{-1}}{M_{N+1} \rho_{N+1} \prod_{\ell=1, \ell \neq i, j}^{k} M_{\ell} \rho_{\ell}}+\sum_{j=1}^{k} \frac{2 M_{j}^{-1} M_{N+1}^{-1}}{\prod_{\ell=1, \ell \neq j}^{k} M_{\ell} \rho_{\ell}} \\
& =\frac{2}{M_{N+1} \prod_{\ell=1}^{k} M_{\ell}}\left(\sum_{i, j=1, i<j}^{k} \frac{1}{\rho_{N+1} \prod_{\ell=1, \ell \neq i, j}^{k} \rho_{\ell}}+\sum_{j=1}^{k} \frac{1}{\prod_{\ell=1, \ell \neq j}^{k} \rho_{\ell}}\right),
\end{aligned}
$$

and (201) follows.

The coefficients $G_{i j}=\partial w_{i} / \partial x_{j}$ are given by

$$
G_{i j}=\frac{1}{M_{N+1} x_{N+1}}+\frac{\delta_{i j}}{M_{i} x_{i}}=c\left(\frac{1}{\rho_{N+1}}+\frac{\delta_{i j}}{\rho_{i}}\right), \quad i, j=1, \ldots, N,
$$

since $x_{i}=\rho_{i} /\left(c M_{i}\right)$. We recall that $w\left(\rho^{\prime}\right)$ is computed in (19).

Lemma 9. It holds for all $\rho_{1}, \ldots, \rho_{N}>0$ satisfying $\rho_{N+1}=1-\sum_{i=1}^{N} \rho_{i}>0$ : 
(i) The matrix $G\left(\rho^{\prime}\right)=\left(G_{i j}\right)$ and its inverse $G^{-1}\left(\rho^{\prime}\right)$ are positive definite.

(ii) $\nabla w\left(\rho^{\prime}\right)=G\left(\rho^{\prime}\right) \nabla x^{\prime}\left(\rho^{\prime}\right)$.

(iii) The elements of the $N \times N$ matrix $d \rho^{\prime} / d x^{\prime}=\left(\partial \rho_{i} / \partial x_{k}\right)$ are bounded by a constant which depends only on the molar masses $M_{i}$.

(iv) The $N \times N$ matrix $B\left(\rho^{\prime}\right)=A_{0}^{-1}\left(\rho^{\prime}\right) G^{-1}\left(\rho^{\prime}\right)$ is symmetric, positive definite, and its elements are uniformly bounded.

Proof. (i) The explicit expression (21) shows that $G\left(\rho^{\prime}\right)$ is symmetric. Since all principal minors $\operatorname{det} G_{k}$ of $G\left(\rho^{\prime}\right)$,

$$
\operatorname{det} G_{k}=\frac{\sum_{i=1}^{k} M_{i} x_{i}+M_{N+1} x_{N+1}}{\left(\prod_{i=1}^{k} M_{i} x_{i}\right) M_{N+1} x_{N+1}}, \quad k=1, \ldots, N,
$$

are positive, Sylvester's criterion implies that $G\left(\rho^{\prime}\right)$ is positive definite. Consequently, also $G^{-1}\left(\rho^{\prime}\right)$ is positive definite.

(ii) We infer from (19) that

$$
\nabla w_{i}=\frac{\nabla x_{i}}{M_{i} x_{i}}+\sum_{j=1}^{N} \frac{\nabla x_{j}}{M_{N+1} x_{N+1}}=\sum_{j=1}^{N} G_{i j} \nabla x_{j}, \quad i=1,2, \ldots, N,
$$

and hence $\nabla w=G\left(\rho^{\prime}\right) \nabla x^{\prime}$.

(iii) By Lemma 6, it follows that

$$
\frac{\partial \rho_{i}}{\partial x_{k}}=c M_{i} \delta_{i k}-c^{2} M_{i} x_{i}\left(M_{k}-M_{N+1}\right), \quad i, k=1, \ldots, N
$$

where $c=1 / \sum_{j=1}^{N+1} M_{j} x_{j}$. The claim follows from the inequalities $0<x_{i}<1$ and the bounds (17).

(iv) We set $G\left(\rho^{\prime}\right)=c K\left(\rho^{\prime}\right)$, where the elements $K_{i j}$ of $K\left(\rho^{\prime}\right)$ are given by $K_{i j}=1 / \rho_{N+1}+$ $\delta_{i j} / \rho_{i}$ for $i, j=1, \ldots, N$. In view of part (i) of the proof, the matrix $K\left(\rho^{\prime}\right)$ is symmetric and positive definite, hence invertible. Then, by Lemma 2.4 in [14, $A_{0}^{-1}\left(\rho^{\prime}\right) K^{-1}\left(\rho^{\prime}\right)$ is symmetric and positive definite and its elements are uniformly bounded. Consequently, the same holds for $B\left(\rho^{\prime}\right)=c^{-1} A_{0}^{-1}\left(\rho^{\prime}\right) K^{-1}\left(\rho^{\prime}\right)$. This ends the proof.

From Lemma 9 follows that

$$
A_{0}^{-1}\left(\rho^{\prime}\right) \nabla x^{\prime}\left(\rho^{\prime}\right)=\left(A_{0}^{-1}\left(\rho^{\prime}\right) G^{-1}\left(\rho^{\prime}\right)\right)\left(G\left(\rho^{\prime}\right) \nabla x^{\prime}\left(\rho^{\prime}\right)\right)=B\left(\rho^{\prime}\right) \nabla w\left(\rho^{\prime}\right) .
$$

We have shown at the end of Section 2.2 that $\rho^{\prime}$ can be interpreted as a function of $w$. Therefore, setting $B(w):=B\left(\rho^{\prime}(w)\right)$, (6) can be written as

$$
\partial_{t} \rho^{\prime}(w)+(u \cdot \nabla) \rho^{\prime}(w)-\operatorname{div}(B(w) \nabla w)=0 .
$$

The boundary conditions are given by

$$
\nabla w_{i} \cdot \nu=0 \quad \text { on } \partial \Omega, t>0, \quad i=1, \ldots, N
$$


since $\nabla \rho_{j} \cdot \nu=0$ on $\partial \Omega$ for all $j$ implies that

$$
\nabla x_{i} \cdot \nu=\nabla \frac{\rho_{i}}{c M_{i}} \cdot \nu=\frac{\nabla \rho_{i} \cdot \nu}{c M_{i}}-\sum_{j=1}^{N+1} \frac{\rho_{i} \nabla \rho_{j} \cdot \nu}{c^{2} M_{i} M_{j}}=0
$$

and thus $\nabla w_{i} \cdot \nu=\left(G\left(\rho^{\prime}\right) \nabla x\right)_{i} \cdot \nu=0$ on $\partial \Omega$.

2.4. Some estimates. We show two results which are needed in the proof of the existence theorem.

Lemma 10. Let $u \in \mathcal{V}$ and $w \in H^{1}(\Omega)$. Then

$$
\int_{\Omega}\left((u \cdot \nabla) \rho^{\prime}(w)\right) \cdot w d z=0 .
$$

Proof. Using $\operatorname{div} u=0$, the characterization (19) of $w_{i}$, and $\rho_{i} / M_{i}=c x_{i}$, we obtain after an integration by parts,

$$
\begin{aligned}
\int_{\Omega}\left((u \cdot \nabla) \rho^{\prime}(w)\right) \cdot w d z & =\sum_{i=1}^{N} \int_{\Omega}\left(u \cdot \nabla \rho_{i}(w)\right) w_{i} d z=-\sum_{i=1}^{N} \int_{\Omega}\left(u \cdot \nabla w_{i}\right) \rho_{i}(w) d z \\
& =-\sum_{i=1}^{N} \int_{\Omega} \rho_{i}(w) u \cdot\left(\frac{\nabla x_{i}}{M_{i} x_{i}}-\frac{\nabla x_{N+1}}{M_{N+1} x_{N+1}}\right) d z \\
& =-\sum_{i=1}^{N} \int_{\Omega} c u \cdot \nabla x_{i} d z+\int_{\Omega} \sum_{i=1}^{N} \rho_{i} \frac{u \cdot \nabla x_{N+1}}{M_{N+1} x_{N+1}} d z
\end{aligned}
$$

Because of $\sum_{i=1}^{N} \rho_{i}=1-\rho_{N+1}$ and $\rho_{N+1} /\left(M_{N+1} x_{N+1}\right)=c$, the last integral equals

$$
\begin{aligned}
\int_{\Omega} \frac{1-\rho_{N+1}}{M_{N+1} x_{N+1}} u \cdot \nabla x_{N+1} d z & =\frac{1}{M_{N+1}} \int_{\Omega} u \cdot \nabla\left(\ln x_{N+1}\right) d z-\int_{\Omega} c u \cdot \nabla x_{N+1} d z \\
& =\sum_{i=1}^{N} \int_{\Omega} c u \cdot \nabla x_{i} d z
\end{aligned}
$$

where we integrated by parts and used div $u=0$ and $x_{N+1}=1-\sum_{i=1}^{N} x_{i}$. This shows the lemma.

In the following, we employ the notation $f(x)=\left(f\left(x_{1}\right), \ldots, f\left(x_{N+1}\right)\right)$ for vectors $x=$ $\left(x_{1}, \ldots, x_{N+1}\right)$ and arbitrary functions $f$.

Lemma 11. Let $w \in H^{1}(\Omega)$. Then there exists a constant $C_{B}>0$, only depending on the coefficients $D_{i j}$ and $M_{i}$ such that

$$
\int_{\Omega} \nabla w: B(w) \nabla w d z \geq C_{B} \int_{\Omega}|\nabla \sqrt{x}|^{2} d z .
$$


Proof. We follow the proof of Lemma 3.2 in [14]. In contrast to that proof, we have to take into account the different molar masses $M_{i}$ which complicates the analysis. First, we claim that

$$
\nabla w: B(w) \nabla w=\nabla s:(-\widetilde{A})^{-1} \nabla x
$$

where $s=\left(\ln x_{1} / M_{1}, \ldots, \ln x_{N+1} / M_{N+1}\right)$ and $\widetilde{A}=\left.A\right|_{\operatorname{im}(A)}$. To prove this claim, we set $r^{\prime}=\left(r_{1}, \ldots, r_{N}\right)^{\top}=B(w) \nabla w \in \mathbb{R}^{N \times d}$ and $r_{N+1}=-\sum_{i=1}^{N} r_{i} \in \mathbb{R}^{d}$. Then, by (19)),

$$
\nabla w: B(w) \nabla w=\sum_{i=1}^{N}\left(\frac{\nabla \ln x_{i}}{M_{i}}-\frac{\nabla \ln x_{N+1}}{M_{N+1}}\right) \cdot r_{i}=\sum_{i=1}^{N+1} \frac{\nabla \ln x_{i}}{M_{i}} \cdot r_{i}=\nabla s: r,
$$

where $r=\left(r^{\prime}, r_{N+1}\right)^{\top}$. By (23),$\nabla x^{\prime}=A_{0} r^{\prime}$, and the definitions (14) and (16) of $A$ and $A_{0}$, respectively, we obtain for $i=1, \ldots, N$,

$$
\nabla x_{i}=\sum_{j=1, j \neq i}^{N}\left(d_{i j}-d_{i, N+1}\right)\left(\rho_{j} r_{i}^{\top}-\rho_{i} r_{j}^{\top}\right)+d_{i, N+1} r_{i}^{\top}=(-A r)_{i}=(-\widetilde{A} r)_{i},
$$

since $\operatorname{im}(A)=(\operatorname{span}(1, \ldots, 1))^{\perp}$ and each column of $r$ is an element of $\operatorname{im}(A)$. Moreover, each column of $\widetilde{A} r$ is also an element of $\operatorname{im}(A)$, so that

$$
(-\widetilde{A} r)_{N+1}=-\sum_{i=1}^{N}(-\widetilde{A} r)_{i}=-\sum_{i=1}^{N} \nabla x_{i}=\nabla x_{N+1} .
$$

Therefore, $\nabla x=-\widetilde{A} r$. It is shown in [14, Lemma 2.2] that $\widetilde{A}$ is invertible. Thus, $r=$ $(-\widetilde{A})^{-1} \nabla x$, and inserting this expression into (26) proves the claim.

Next, we introduce the symmetric matrix $\widetilde{A}_{S}=P^{-1 / 2} \widetilde{A} P^{1 / 2}$, where

$$
P^{1 / 2}=\operatorname{diag}\left(\left(M_{1} x_{1}\right)^{1 / 2}, \ldots,\left(M_{N+1} x_{N+1}\right)^{1 / 2}\right) .
$$

Then $\left(-\widetilde{A}_{S}\right)^{-1}=P^{-1 / 2}(-\widetilde{A})^{-1} P^{1 / 2}$. Arguing similarly as in [14, Lemma 2.2], we find that $\left(-\widetilde{A}_{S}\right)^{-1}$ is a self-adjoint endomorphism whose smallest eigenvalue is bounded from below by some positive constant, say $C_{0}>0$, which depends only on $\left(D_{i j}\right)$. This gives

$$
\begin{aligned}
\nabla w: & B(w) \nabla w=\nabla s:(-\widetilde{A})^{-1} \nabla x \\
= & 4 \nabla \sqrt{x}: \operatorname{diag}\left(M_{1}^{-1} x_{1}^{-1 / 2}, \ldots, M_{N+1}^{-1} x_{N+1}^{-1 / 2}\right)(-\widetilde{A})^{-1} \operatorname{diag}\left(x_{1}^{1 / 2}, \ldots, x_{N+1}^{1 / 2}\right) \nabla \sqrt{x} \\
= & 4 \nabla \sqrt{x}:\left(\operatorname{diag}\left(M_{1}^{-1} x_{1}^{-1 / 2}, \ldots, M_{N+1}^{-1} x_{N+1}^{-1 / 2}\right) P^{1 / 2}\right)\left(P^{-1 / 2}(-\widetilde{A})^{-1} P^{1 / 2}\right) \\
& \times\left(P^{-1 / 2} \operatorname{diag}\left(x_{1}^{1 / 2}, \ldots, x_{N+1}^{1 / 2}\right)\right) \nabla \sqrt{x} \\
= & 4 \nabla \sqrt{x}: \operatorname{diag}\left(M_{1}^{-1 / 2}, \ldots, M_{N+1}^{-1 / 2}\right)\left(-\widetilde{A}_{S}\right)^{-1} \operatorname{diag}\left(M_{1}^{-1 / 2}, \ldots, M_{N+1}^{-1 / 2}\right) \nabla \sqrt{x} \\
\geq & C_{0}\left|\operatorname{diag}\left(M_{1}^{-1 / 2}, \ldots, M_{N+1}^{-1 / 2}\right) \nabla \sqrt{x}\right|^{2} \\
\geq & C_{B}|\nabla \sqrt{x}|^{2}
\end{aligned}
$$

where $C_{B}=C_{0}\left(\max _{1 \leq i \leq N+1} M_{i}\right)^{-1 / 2}$. 


\section{Proof of Theorem 1}

We say that $(u, \rho)$ is a weak solution to (11)-(4) if for any $v \in C_{0}^{\infty}\left(\Omega \times[0, T) ; \mathbb{R}^{d}\right)$ with $\operatorname{div} v=0$,

$$
\begin{gathered}
-\int_{0}^{T} \int_{\Omega} u \cdot \partial_{t} v d z d t+\int_{0}^{T} \int_{\Omega}((u \cdot \nabla) u) \cdot v d z d t+\int_{0}^{T} \int_{\Omega} \nabla u: \nabla v d z d t \\
=\int_{0}^{T}\langle f, v\rangle d t+\int_{\Omega} u^{0} \cdot v(\cdot, 0) d z
\end{gathered}
$$

where $\langle\cdot, \cdot\rangle$ denotes the duality pairing between $\mathcal{V}^{\prime}$ and $\mathcal{V}$; and if for any $q \in C_{0}^{\infty}(\bar{\Omega} \times$ $\left.[0, T) ; \mathbb{R}^{N}\right)$ with $\left.\nabla q \cdot \nu\right|_{\partial \Omega}=0$

$$
\begin{gathered}
-\int_{0}^{T} \int_{\Omega} \rho^{\prime} \cdot \partial_{t} q d z d t+\int_{0}^{T} \int_{\Omega} \nabla q: A_{0}^{-1}\left(\rho^{\prime}\right) \nabla x^{\prime}\left(\rho^{\prime}\right) d z d t+\int_{0}^{T} \int_{\Omega}\left((u \cdot \nabla) \rho^{\prime}\right) \cdot q d z d t \\
=\int_{\Omega}\left(\rho^{0}\right)^{\prime} \cdot q(\cdot, 0) d z .
\end{gathered}
$$

The proof of Theorem 1 is divided into several steps.

3.1. Approximate problem. Let $M \in \mathbb{N}$ and set $\tau=T / M$. Let $k \in\{1, \ldots, M\}$. Given $\left(u^{k-1}, w^{k-1}\right) \in \mathcal{V} \times L^{\infty}\left(\Omega ; \mathbb{R}^{N}\right)$, we solve a regularized approximate problem for (1)-(3) : For any $v \in \mathcal{V}$ and $q \in \widetilde{H}^{2}\left(\Omega ; \mathbb{R}^{N}\right)$ :

$$
\begin{aligned}
& \int_{\Omega} \frac{u^{k}-u^{k-1}}{\tau} \cdot v d z+\int_{\Omega}\left(\left(u^{k-1} \cdot \nabla\right) u^{k}\right) \cdot v d z+\int_{\Omega} \nabla u^{k}: \nabla v d z=\int_{\Omega} f^{k} \cdot v d z \\
& \int_{\Omega} \frac{\rho^{\prime}\left(w^{k}\right)-\rho^{\prime}\left(w^{k-1}\right)}{\tau} \cdot q d z+\int_{\Omega} \nabla q: A_{0}^{-1}\left(\rho^{\prime}\left(w^{k}\right)\right) \nabla x^{\prime}\left(\rho^{\prime}\left(w^{k}\right)\right) d z \\
& \quad+\int_{\Omega}\left(\left(u^{k} \cdot \nabla\right) \rho^{\prime}\left(w^{k}\right)\right) \cdot q d z+\varepsilon \int_{\Omega}\left(\Delta w^{k} \cdot \Delta q+w^{k} \cdot q\right) d z=0
\end{aligned}
$$

where $f^{k}=\tau^{-1} \int_{(k-1) \tau}^{k \tau} f(\cdot, t) d t \in \mathcal{V}^{\prime}$ and $\rho^{\prime}\left(w^{k}\right)$ is defined in Corollary 7 . Because of (23) , equation (30) is equivalent to

$$
\begin{aligned}
& \int_{\Omega} \frac{\rho^{\prime}\left(w^{k}\right)-\rho^{\prime}\left(w^{k-1}\right)}{\tau} \cdot q d z+\int_{\Omega} \nabla q: B\left(w^{k}\right) \nabla w^{k} d z \\
& \quad+\int_{\Omega}\left(\left(u^{k} \cdot \nabla\right) \rho^{\prime}\left(w^{k}\right)\right) \cdot q d z+\varepsilon \int_{\Omega}\left(\Delta w^{k} \cdot \Delta q^{k}+w^{k} \cdot q\right) d z=0,
\end{aligned}
$$

Define for $0<\eta<1$ the space of bounded, strictly positive functions

$$
Y_{\eta}=\left\{q=\left(q_{1}, \ldots, q_{N}\right) \in L^{\infty}\left(\Omega ; \mathbb{R}^{N}\right): q_{i} \geq \eta \text { for } i=1, \ldots N, q_{N+1}=1-\sum_{i=1}^{N} q_{i} \geq \eta\right\} .
$$

Lemma 12. Let $\eta^{k-1} \in(0,1)$ and $\left(u^{k-1}, \rho^{k-1}\right) \in \mathcal{V} \times Y_{\eta^{k-1}}$ with $\rho^{k-1}=\rho^{\prime}\left(w^{k-1}\right)$. Then there exist $\eta^{k} \in(0,1)$ and $\left(u^{k}, w^{k}\right) \in \mathcal{V} \times \widetilde{H}^{2}\left(\Omega ; \mathbb{R}^{N}\right)$ which solves (29)-(30) satisfying $\rho^{\prime}\left(w^{k}\right) \in Y_{\eta^{k}}$. 
Proof. Step 1. By standard theory of the incompressible Navier-Stokes equations [18], there exists a unique solution $u \in \mathcal{V}$ to

$$
a_{1}(u, v)=F_{1}(v) \text { for } v \in \mathcal{V}
$$

where for $u, v \in \mathcal{V}$,

$$
\begin{aligned}
a_{1}(u, v) & =\frac{1}{\tau} \int_{\Omega} u \cdot v d z+\int_{\Omega}\left(\left(u^{k-1} \cdot \nabla\right) u\right) \cdot v d z+\int_{\Omega} \nabla u: \nabla v d z, \\
F_{1}(v) & =\frac{1}{\tau} \int_{\Omega} u^{k-1} \cdot v d z+\int_{\Omega} f^{k} \cdot v d z .
\end{aligned}
$$

Indeed, since $H^{1}(\Omega) \hookrightarrow L^{4}(\Omega)$ for $d \leq 4$, we have for some (generic) constant $C>0$,

$$
\left|\int_{\Omega}\left(\left(u^{k-1} \cdot \nabla\right) u\right) \cdot v d z\right| \leq\left\|u^{k-1}\right\|_{L^{4}(\Omega)}\|\nabla u\|_{L^{2}(\Omega)}\|v\|_{L^{4}(\Omega)} \leq C\|u\|_{H^{1}(\Omega)}\|v\|_{H^{1}(\Omega)},
$$

and using $\operatorname{div} u^{k-1}=0$, it follows that

$$
a_{1}(u, u)=\frac{1}{\tau} \int_{\Omega}|u|^{2} d z+\int_{\Omega}\|\nabla u\|^{2} d z \geq C\|u\|_{H^{1}(\Omega)}^{2} .
$$

Thus, $a_{1}(\cdot, \cdot)$ is a bounded, coercive bilinear form on $\mathcal{V}$ and $F_{1} \in \mathcal{V}^{\prime}$. By Lax-Milgram's lemma, there exists a unique solution $u \in \mathcal{V}$ to (32).

Step 2. Let $u \in \mathcal{V}$ be the unique solution to (32) and let $\bar{w} \in L^{\infty}\left(\Omega ; \mathbb{R}^{N}\right)$. Let $\sigma \in[0,1]$. We prove that there exists a unique $w \in \widetilde{H}^{2}\left(\Omega ; \mathbb{R}^{N}\right)$ to

$$
a_{2}(w, q)=F_{2}(q) \quad \text { for } q \in \widetilde{H}^{2}\left(\Omega ; \mathbb{R}^{N}\right),
$$

where for $w, q \in \widetilde{H}^{2}\left(\Omega ; \mathbb{R}^{N}\right)$,

$$
\begin{aligned}
a_{2}(w, q) & =\varepsilon \int_{\Omega}(\Delta w \cdot \Delta q+w \cdot q) d z+\int_{\Omega} \nabla q: B(\bar{w}) \nabla w d z \\
F_{2}(q) & =-\frac{\sigma}{\tau} \int_{\Omega}\left(\rho^{\prime}(\bar{w})-\rho^{k-1}\right) \cdot q d z+\sigma \int_{\Omega}((u \cdot \nabla) q) \cdot \rho^{\prime}(\bar{w}) d z .
\end{aligned}
$$

We infer from Lemma 9 (iv) that $a_{2}(\cdot, \cdot)$ is a bounded bilinear form on $\widetilde{H}^{2}\left(\Omega ; \mathbb{R}^{N}\right)$, and from the positive definiteness of $B(\bar{w})$ (see also Lemma 9 (iv)) follows that

$$
a_{2}(w, w) \geq \varepsilon \int_{\Omega}\left(|\Delta w|^{2}+|w|^{2}\right) d z \geq C\|w\|_{H^{2}(\Omega)}^{2} .
$$

Since $\rho^{\prime}(\bar{w})$ is a bounded function, by Corollary 7 , we infer that $F_{2}$ is bounded on $\widetilde{H}^{2}\left(\Omega ; \mathbb{R}^{N}\right)$. Then the Lax-Milgram lemma provides the existence of a unique solution $w \in \widetilde{H}^{2}\left(\Omega ; \mathbb{R}^{N}\right)$ to (33).

Step 3. This defines the fixed-point mapping $S: L^{\infty}\left(\Omega ; \mathbb{R}^{N}\right) \times[0,1] \rightarrow L^{\infty}\left(\Omega ; \mathbb{R}^{N}\right)$, $S(\bar{w}, \sigma)=w$, where $w$ solves $(\underline{33})$. By construction, $S(\bar{w}, 0)=0$ for all $w \in L^{\infty}\left(\Omega ; \mathbb{R}^{N}\right)$. Since the embedding $H^{2}(\Omega) \hookrightarrow L^{\infty}(\Omega)$ is compact, standard arguments show that $S$ is continuous and compact. It remains to prove that there exists a constant $C>0$ such that $\|w\|_{L^{\infty}(\Omega)} \leq C$ for all $(w, \sigma) \in L^{\infty}\left(\Omega ; \mathbb{R}^{N}\right) \times[0,1]$ satisfying $w=S(w, \sigma)$. 
Let $w \in L^{\infty}\left(\Omega ; \mathbb{R}^{N}\right)$ be such a fixed point. Then it solves (33) with $\bar{w}$ replaced by $w$. Taking $w \in \widetilde{H}^{2}\left(\Omega ; \mathbb{R}^{N}\right)$ as a test function, it follows from Lemma 10 that

$$
\frac{\sigma}{\tau} \int_{\Omega}\left(\rho^{\prime}(w)-\rho^{\prime}\left(w^{k-1}\right)\right) \cdot w d z+\int_{\Omega} \nabla w: B(w) \nabla w d z+\varepsilon \int_{\Omega}\left(|\Delta w|^{2}+|w|^{2}\right) d z=0 .
$$

By Lemma 8, the entropy density $h$, defined in (7), is convex. This implies that $h\left(\rho^{\prime}(w)\right)-$ $h\left(\rho^{k-1}\right) \leq\left(d h / d \rho^{\prime}\right) \cdot\left(\rho^{\prime}(w)-\rho^{k-1}\right)=w \cdot\left(\rho^{\prime}(w)-\rho^{k-1}\right)$ (see Lemma 44). We infer from the positive definiteness of $B(w)$ (see Lemma 9 (iv)) that

$$
\sigma \int_{\Omega} h\left(\rho^{\prime}(w)\right) d z+\varepsilon \tau \int_{\Omega}\left(|\Delta w|^{2}+|w|^{2}\right) d z \leq \sigma \int_{\Omega} h\left(\rho^{k-1}\right) d z .
$$

This yields the desired uniform $H^{2}$ bound and hence uniform $L^{\infty}$ bound for $w$. By the Leray-Schauder fixed-point theorem, there exists a solution $w \in \widetilde{H}^{2}\left(\Omega ; \mathbb{R}^{N}\right)$ to (31). According to Corollary 7 , we can define $\rho_{1}(w), \ldots, \rho_{N}(w)>0$ satisfying $\rho_{N+1}(w):=$ $1-\sum_{i=1} \rho_{i}(w)>0$, and we set $\eta^{k}=\min _{1 \leq i \leq N+1} \operatorname{essinf}_{\Omega} \rho_{i}(w)>0$. Then, by construction, $\rho^{\prime}(w) \in Y_{\eta^{k}}$.

3.2. Uniform estimates. Let $u^{0} \in \mathcal{H}$ and $\rho^{0}=\left(\rho_{1}^{0}, \ldots, \rho_{N+1}^{0}\right)$ satisfing $\rho_{i}^{0} \geq 0$ for $i=$ $1, \ldots, N+1$ and $\sum_{i=1}^{N+1} \rho_{i}^{0}=1$. We regularize $u^{0}$ by $u_{\tau}^{0}$ which is the weak solution to $u_{\tau}^{0}-\tau \Delta u_{\tau}^{0}=u^{0}$ in $\Omega$ and $u_{\tau}^{0}=0$ on $\partial \Omega$. Then it holds that

$$
\left\|u_{\tau}^{0}\right\|_{L^{2}(\Omega)}^{2}+2 \tau\left\|\nabla u_{\tau}^{0}\right\|_{L^{2}(\Omega)}^{2} \leq\left\|u^{0}\right\|_{L^{2}(\Omega)}^{2}
$$

and (at least for a subsequence) $u_{\tau}^{0} \rightarrow u^{0}$ weakly in $L^{2}\left(\Omega ; \mathbb{R}^{d}\right)$. Let $0<\eta^{0} \leq 1 /(2(N+1))$ and define

$$
\rho_{i}^{\eta^{0}}=\frac{\rho_{i}^{0}+2 \eta^{0}}{1+2 \eta^{0}(N+1)}, \quad i=1, \ldots, N+1
$$

Then $\rho_{i}^{\eta^{0}} \geq \eta^{0}$ for all $i=1, \ldots, N+1$ and $\sum_{i=1}^{N+1} \rho_{i}^{\eta^{0}}=1$. Finally, let $w^{0} \in L^{\infty}\left(\Omega ; \mathbb{R}^{N}\right)$ be defined by (19). Applying Lemma 12 iteratively, we obtain a sequence of approximate solutions $\left(u^{k}, w^{k}\right) \in \mathcal{V} \times \widetilde{H}^{2}\left(\Omega ; \mathbb{R}^{N}\right)$ to (29)- For the following, we set $\rho^{k}=\rho^{\prime}\left(w^{k}\right)$ for $k \geq 0$, slightly abusing our notation.

Lemma 13. For any $1 \leq k \leq M$ and sufficiently small $\eta^{0}>0$, it holds that

$$
\int_{\Omega} h\left(\rho^{k}\right) d z+C_{B} \tau \sum_{j=1}^{k}\left\|\nabla \sqrt{x\left(\rho^{j}\right)}\right\|_{L^{2}(\Omega)}^{2}+\varepsilon \tau \sum_{j=1}^{k} \int_{\Omega}\left(\left|\Delta w^{j}\right|^{2}+\left|w^{j}\right|^{2}\right) d z \leq \int_{\Omega} h\left(\rho^{0}\right) d z+1,
$$

where $\sqrt{x\left(\rho^{j}\right)}=\left(\sqrt{x_{1}\left(\rho^{j}\right)}, \ldots, \sqrt{x_{N+1}\left(\rho^{j}\right)}\right), x_{i}\left(\rho^{j}\right)=\rho_{i}^{j} /\left(c M_{i}\right)$ for $i=1, \ldots, N+1$, $c=\sum_{k=1}^{N+1} \rho_{k}^{j} / M_{k}$, and $C_{B}>0$ is obtained from Lemma 11 . 
Proof. The proof of (34) is standard and we refer to [18, Section III.4.3] for a proof. Lemma 11 and Step 3 of the proof of Lemma 12 imply after summation over $j=1, \ldots, k$ that

$$
\int_{\Omega} h\left(\rho^{k}\right) d z+C_{B} \tau \sum_{j=1}^{k}\left\|\sqrt{x\left(\rho^{j}\right)}\right\|_{L^{2}(\Omega)}^{2}+\varepsilon \tau \sum_{j=1}^{k} \int_{\Omega}\left(\left|\Delta w^{j}\right|^{2}+\left|w^{j}\right|^{2}\right) d z \leq \int_{\Omega} h\left(\rho^{\eta^{0}}\right) d z .
$$

By dominated convergence,

$$
\lim _{\eta^{0} \rightarrow 0} \int_{\Omega} h\left(\rho^{\eta^{0}}\right) d z=\int_{\Omega} h\left(\rho^{0}\right) d z
$$

and hence, for sufficiently small $\eta^{0}>0$,

$$
\int_{\Omega} h\left(\rho^{\eta^{0}}\right) d z \leq \int_{\Omega} h\left(\rho^{0}\right) d z+1
$$

This proves (35).

Lemma 14. It holds that

$$
\begin{array}{r}
\tau \sum_{k=1}^{M}\left\|\frac{u^{k}-u^{k-1}}{\tau}\right\|_{\mathcal{V}_{2}^{\prime}}^{2} \leq C\left(u^{0}, f\right), \\
\tau \sum_{k=1}^{M}\left\|\nabla x\left(\rho^{k}\right)\right\|_{L^{2}(\Omega)}^{2}+\tau \sum_{k=1}^{M}\left\|\nabla \rho^{k}\right\|_{L^{2}(\Omega)}^{2}+\tau \sum_{k=1}^{M}\left\|\frac{\rho^{k}-\rho^{k-1}}{\tau}\right\|_{\widetilde{H}^{2}(\Omega)^{\prime}}^{2} \leq C\left(u^{0}, \rho^{0}, f\right),
\end{array}
$$

where $\mathcal{V}_{2}^{\prime}$ is the dual space of $\mathcal{V}_{2}$, defined in (5).

Proof. Again, estimate (36) is standard; see [18, Section III.4.3]. Since $x_{i}\left(\rho^{k}\right)=\rho_{i}^{k} /\left(c M_{i}\right)$ with $c=\sum_{k=1}^{N+1} \rho_{k} / M_{k}$ is bounded by one, we find that

$$
\left\|\nabla x\left(\rho^{k}\right)\right\|_{L^{2}(\Omega)} \leq 2\left\|\sqrt{x\left(\rho^{k}\right)}\right\|_{L^{2}(\Omega)}\left\|\nabla \sqrt{x\left(\rho^{k}\right)}\right\|_{L^{2}(\Omega)} \leq 2\left\|\nabla \sqrt{x\left(\rho^{k}\right)}\right\|_{L^{2}(\Omega)} .
$$

Thus, by (35),

$$
\tau \sum_{k=1}^{M}\left\|\nabla x\left(\rho^{k}\right)\right\|_{L^{2}(\Omega)}^{2} \leq C\left(\rho^{0}\right) .
$$

Then it follows from Lemma 9 (iii) that $\left|\nabla \rho^{k}\right| \leq C\left|\nabla x^{\prime}\left(\rho^{k}\right)\right|$. Hence,

$$
\tau \sum_{k=1}^{M}\left\|\nabla \rho^{k}\right\|_{L^{2}(\Omega)}^{2} \leq C \tau \sum_{k=1}^{M}\left\|\nabla x^{\prime}\left(\rho^{k}\right)\right\|_{L^{2}(\Omega)}^{2} \leq C\left(\rho^{0}\right) .
$$

We deduce from (30), the boundedness of the elements of $A_{0}^{-1}\left(\rho^{k}\right)$ (see Lemma 3 ), and the uniform estimate for $u_{k}$ in $L^{2}$ (see (34)) that for $q \in \widetilde{H}^{2}\left(\Omega ; \mathbb{R}^{N}\right)$,

$$
\begin{aligned}
& \left|\frac{1}{\tau} \int_{\Omega}\left(\rho^{k}-\rho^{k-1}\right) \cdot q d z\right| \\
& \leq\left\|A_{0}^{-1}\left(\rho^{k}\right)\right\|_{L^{\infty}(\Omega)}\left\|\nabla x^{\prime}\left(\rho^{k}\right)\right\|_{L^{2}(\Omega)}\|q\|_{L^{2}(\Omega)} \\
& \quad+\left\|u^{k}\right\|_{L^{2}(\Omega)}\left\|\nabla \rho^{k}\right\|_{L^{2}(\Omega)}\|q\|_{L^{\infty}(\Omega)}+\varepsilon\left(\left\|\Delta w^{k}\right\|_{L^{2}(\Omega)}+\left\|w^{k}\right\|_{L^{2}(\Omega)}\right)\|q\|_{L^{2}(\Omega)}
\end{aligned}
$$




$$
\leq C\left(u^{0}, f\right)\left(\left\|\nabla x^{\prime}\left(\rho^{k}\right)\right\|_{L^{2}(\Omega)}+\left\|\nabla \rho^{k}\right\|_{L^{2}(\Omega)}+\varepsilon\left\|w^{k}\right\|_{H^{2}(\Omega)}\right)\|q\|_{H^{2}(\Omega)} .
$$

Taking into account the above uniform estimates for $\nabla x^{\prime}\left(\rho^{k}\right)$ and $\nabla \rho^{k}$ in $L^{2}$ and the estimate (35) for $\sqrt{\varepsilon} w^{k}$ in $H^{2}$, it follows that

$$
\begin{aligned}
\tau \sum_{k=1}^{M}\left\|\frac{\rho^{k}-\rho^{k-1}}{\tau}\right\|_{\widetilde{H}^{2}(\Omega)^{\prime}}^{2} & \leq C\left(u^{0}, f\right) \tau \sum_{k=1}^{M}\left(\left\|\nabla x^{\prime}\left(\rho^{k}\right)\right\|_{L^{2}(\Omega)}^{2}+\left\|\nabla \rho^{k}\right\|_{L^{2}(\Omega)}^{2}+\varepsilon^{2}\left\|w^{k}\right\|_{H^{2}(\Omega)}^{2}\right) \\
& \leq C\left(u^{0}, \rho^{0}, f\right) .
\end{aligned}
$$

This ends the proof.

3.3. Proof of Theorem 1. Define the piecewise constant function $u^{(\tau)}(x, t)=u^{k}(x)$, its time shift $\left(\pi_{\tau} u^{(\tau)}\right)(x, t)=u^{k-1}(x)$ and the difference quotient

$$
\partial_{t}^{\tau} u^{(\tau)}(x, t)=\frac{u^{k}(x)-u^{k-1}(x)}{\tau}
$$

for $x \in \Omega,(k-1) \tau<t \leq k \tau, k=1, \ldots, M$. Similarly, we define $f^{(\tau)}, w^{(\tau)}, \rho^{(\tau)}$, and $\partial_{t}^{\tau} \rho^{(\tau)}$. Lemmas 13 and 14 imply immediately the following uniform estimates:

$$
\begin{aligned}
\left\|u^{(\tau)}\right\|_{L^{\infty}\left(0, T ; L^{2}(\Omega)\right)}+\left\|u^{(\tau)}\right\|_{L^{2}\left(0, T ; H^{1}(\Omega)\right)}+\left\|\partial_{t}^{\tau} u^{(\tau)}\right\|_{L^{2}\left(0, T ; \mathcal{V}_{2}^{\prime}\right)} & \leq C, \\
\tau^{-1}\left\|\pi_{\tau} u^{(\tau)}-u^{(\tau)}\right\|_{L^{2}\left(0, T ; L^{2}(\Omega)\right)} & \leq C, \\
\left\|x^{\prime}\left(\rho^{(\tau)}\right)\right\|_{L^{\infty}\left(0, T ; L^{\infty}(\Omega)\right)}+\left\|x^{\prime}\left(\rho^{(\tau)}\right)\right\|_{L^{2}\left(0, T ; H^{1}(\Omega)\right)} & \leq C, \\
\left\|\rho^{(\tau)}\right\|_{L^{\infty}\left(0, T ; L^{\infty}(\Omega)\right)}+\left\|\rho^{(\tau)}\right\|_{L^{2}\left(0, T ; H^{1}(\Omega)\right)}+\left\|\partial_{t}^{\tau} \rho^{(\tau)}\right\|_{L^{2}\left(0, T ; \widetilde{H}^{2}(\Omega)^{\prime}\right)} & \leq C, \\
\sqrt{\varepsilon}\left\|w^{(\tau)}\right\|_{L^{2}\left(0, T ; H^{2}(\Omega)\right)} & \leq C .
\end{aligned}
$$

The weak formulation $(\underline{29})-(\underline{30})$ can be written for any $v \in C_{0}^{\infty}\left(\Omega \times[0, T) ; \mathbb{R}^{d}\right)$ with $\operatorname{div} v=0$ and any $q \in C_{0}^{\infty}\left(\bar{\Omega} \times[0, T) ; \mathbb{R}^{N}\right)$ with $\left.\nabla q \cdot \nu\right|_{\partial \Omega}=0$ as follows:

$$
\begin{aligned}
& \int_{0}^{T} \int_{\Omega} \partial_{t}^{\tau} u^{(\tau)} \cdot v d z d t+\int_{0}^{T} \int_{\Omega} \nabla u^{(\tau)}: \nabla v d z d t+\int_{0}^{T} \int_{\Omega}\left(\left(\pi_{\tau} u^{(\tau)} \cdot \nabla\right) u^{(\tau)}\right) \cdot v d z d t \\
& (43) \quad=\int_{0}^{T} \int_{\Omega} f^{(\tau)} \cdot v d z d t \\
& \int_{0}^{T} \int_{\Omega} \partial_{t}^{\tau} \rho^{(\tau)} \cdot q d z d t+\int_{0}^{T} \int_{\Omega} \nabla q: A_{0}^{-1}\left(\rho^{(\tau)}\right) \nabla x^{\prime}\left(\rho^{(\tau)}\right) d z d t+\int_{0}^{T} \int_{\Omega}\left(\left(u^{(\tau)} \cdot \nabla\right) \rho^{(\tau)}\right) \cdot q d z d t \\
& (44) \quad=-\varepsilon \int_{0}^{T} \int_{\Omega}\left(\Delta w^{(\tau)} \cdot \Delta q+w^{(\tau)} \cdot q\right) d z d t .
\end{aligned}
$$

Estimates (38) for $\left(u^{(\tau)}\right)$ and (41) for $\left(\rho^{(\tau)}\right)$ allow us to apply Aubin's lemma in the version of [8] which yields the existence of subsequences of $\left(u^{(\tau)}\right)$ and $\left(\rho^{(\tau)}\right)$ (not relabeled) such that, as $(\varepsilon, \tau) \rightarrow 0$,

$$
u^{(\tau)} \rightarrow u, \quad \rho^{(\tau)} \rightarrow \rho^{\prime} \quad \text { strongly in } L^{2}\left(0, T ; L^{2}(\Omega)\right) .
$$


Consequently, by (39),

$$
\left\|\pi_{\tau} u^{(\tau)}-u\right\|_{L^{2}\left(0, T ; L^{2}(\Omega)\right)} \leq\left\|\pi_{\tau} u^{(\tau)}-u^{(\tau)}\right\|_{L^{2}\left(0, T ; L^{2}(\Omega)\right)}+\left\|u^{(\tau)}-u\right\|_{L^{2}\left(0, T ; L^{2}(\Omega)\right)} \rightarrow 0
$$

and $\left(\pi_{\tau} u^{(\tau)} \cdot \nabla\right) u^{(\tau)} \rightarrow(u \cdot \nabla) u$ weakly in $L^{1}\left(0, T ; L^{1}(\Omega)\right)$. Furthermore, the strong convergence of $\left(\rho^{(\tau)}\right)$ and the boundedness of the elements of $A_{0}^{-1}$ and $x^{\prime}$ yield $A_{0}^{-1}\left(\rho^{(\tau)}\right) \rightarrow$ $A_{0}^{-1}\left(\rho^{\prime}\right), x^{\prime}\left(\rho^{(\tau)}\right) \rightarrow x^{\prime}\left(\rho^{\prime}\right)$ strongly in $L^{p}\left(0, T ; L^{p}(\Omega)\right)$ for any $p<\infty$. Together with the weak convergence (again up to a subsequence) of $\left(\nabla x^{\prime}\left(\rho^{(\tau)}\right)\right)$, we infer that

$$
\nabla x^{\prime}\left(\rho^{(\tau)}\right) \rightarrow \nabla x^{\prime}(\rho) \quad \text { weakly in } L^{2}\left(0, T ; L^{2}(\Omega)\right) .
$$

Finally, we note that $f^{(\tau)} \rightarrow f$ strongly in $L^{2}\left(0, T ; \mathcal{V}^{\prime}\right)$ (see [18, Lemma III.4.9]) and $\varepsilon w^{(\tau)} \rightarrow 0$ strongly in $L^{2}\left(0, T ; H^{2}(\Omega)\right)$ as $(\varepsilon, \tau) \rightarrow 0$. These convergences are sufficient to pass to the limit $(\varepsilon, \tau) \rightarrow 0$ in (43)-(44) yielding a global solution $\left(u, \rho^{\prime}\right)$ to (27)-(28). In view of the a priori estimates uniform in $\eta^{0}$ and the finiteness of the initial entropy, we can perform the limit $\eta^{0} \rightarrow 0$ and hence conclude the existence result for general initial data. The theorem is proved.

\section{Proof of Theorem 2}

Let $\left(u^{k}, w^{k}\right)$ be a solution to (29) and (31). First, we prove $L^{1}$ bounds for $\rho_{i}^{k}=\rho_{i}\left(w^{k}\right)$ and $c^{k}=\sum_{i=1}^{N+1} \rho_{i}^{k} / M_{k}$.

Lemma 15 (Uniform $L^{1}$ norms for $\rho^{k}$ ). There exist constants $\gamma_{0}>0$, depending on $\rho^{0}$, and $\varepsilon_{0}>0$ such that for all $0<\gamma<\min \left\{1, \gamma_{0}\right\}$ and $0<\varepsilon<\varepsilon_{0}$,

$$
\begin{aligned}
\left|\left\|\rho_{i}^{k}\right\|_{L^{1}(\Omega)}-\left\|\rho_{i}^{0}\right\|_{L^{1}(\Omega)}\right| & \leq \gamma\left\|\rho_{i}^{0}\right\|_{L^{1}(\Omega)}, \quad i=1, \ldots, N, \\
\left|\left\|\rho_{N+1}^{k}\right\|_{L^{1}(\Omega)}-\left\|\rho_{N+1}^{0}\right\|_{L^{1}(\Omega)}\right| & \leq \gamma \sum_{I=1}^{N}\left\|\rho_{i}^{0}\right\|_{L^{1}(\Omega)} .
\end{aligned}
$$

Furthermore, $\left\|\rho_{N+1}^{k}\right\|_{L^{1}(\Omega)} \geq \frac{1}{2}\left\|\rho_{N+1}^{0}\right\|_{L^{1}(\Omega)}>0$.

Proof. The proof is similar to the proof of Lemma 4.1 in [14. The main difference is that the entropy differs from that of [14] which makes some changes necessary. We recall that $\tau=T / M$ with $T>0$ and $M \in \mathbb{N}$. Using the test function $q=e_{i}$ in (31), where $e_{i}$ is the $i$ th unit vector of $\mathbb{R}^{N}$, and observing that

$$
\int_{\Omega}\left(\left(u^{k} \cdot \nabla\right) \rho^{\prime}\left(w^{k}\right)\right) \cdot e_{i} d z=-\int_{\Omega} \operatorname{div}\left(u^{k}\right) \rho_{i}\left(w^{k}\right) d z=0,
$$

we have

$$
\int_{\Omega} \rho_{i}^{k} d z=\int_{\Omega} \rho_{i}^{k-1}-\varepsilon \tau \int_{\Omega} w_{i}^{k} d z, \quad i=1, \ldots, N
$$

Solving this recursion, we deduce that

$$
\int_{\Omega} \rho_{i}^{k} d z=\int_{\Omega} \rho_{i}^{0} d z-\varepsilon \tau \sum_{j=1}^{k} \int_{\Omega} w_{i}^{j} d z, \quad i=1, \ldots, N .
$$


Thus, we need to bound the $L^{1}$ norm of $w_{i}^{j}$. Recalling that $H\left(\rho^{k}\right)=\int_{\Omega} h\left(\rho^{\prime}\left(w^{k}\right)\right) d z$, we infer from Step 3 of the proof of Lemma 12 that

$$
H\left(\rho^{k}\right)+\varepsilon \tau \int_{\Omega}\left|w_{i}^{k}\right|^{2} d z \leq H\left(\rho^{k-1}\right)
$$

or, solving the recursion,

$$
H\left(\rho^{k}\right)+\varepsilon \tau \sum_{j=1}^{k} \int_{\Omega}\left|w_{i}^{j}\right|^{2} d z \leq H\left(\rho^{0}\right) .
$$

It follows from the definition of the entropy and estimate (17) that the entropy can be bounded from below:

$$
H\left(\rho^{k}\right)=\int_{\Omega} c^{k} \sum_{j=1}^{N+1}\left(x_{i}^{k}\left(\ln x_{i}^{k}-1\right)+1\right)-N \int_{\Omega} c^{k} d z \geq-C_{1}:=-N \operatorname{meas}(\Omega) M_{*}^{-1},
$$

where $c^{k}=\sum_{i=1}^{N+1} \rho_{i}^{k} / M_{i}, x_{i}^{k}=\rho_{i}^{k} /\left(c^{k} M_{i}\right)$, and $M_{*}=\min _{1 \leq i \leq N+1} M_{i}$. Therefore, (48) implies that

$$
\varepsilon \tau \sum_{j=1}^{k} \int_{\Omega}\left|w_{i}^{j}\right|^{2} d z \leq H\left(\rho^{0}\right)-H\left(\rho^{k}\right) \leq H\left(\rho^{0}\right)+C_{1} .
$$

The $L^{1}$ norm of $w_{i}^{k}$ can be estimated by its $L^{2}$ norm by applying the Cauchy-Schwarz inequality:

$$
\begin{aligned}
\varepsilon \tau \sum_{j=1}^{k} \int_{\Omega}\left|w_{i}^{j}\right| d z & \leq \varepsilon \tau \sqrt{\operatorname{meas}(\Omega)} \sum_{j=1}^{k}\left\|w_{i}^{j}\right\|_{L^{2}(\Omega)} \leq \varepsilon \tau \sqrt{k \operatorname{meas}(\Omega)}\left(\sum_{j=1}^{k}\left\|w_{i}^{j}\right\|_{L^{2}(\Omega)}^{2}\right)^{1 / 2} \\
& =\sqrt{\varepsilon \tau k \operatorname{meas}(\Omega)}\left(\varepsilon \tau \sum_{j=1}^{k}\left\|w_{i}^{j}\right\|_{L^{2}(\Omega)}^{2}\right)^{1 / 2} \leq \sqrt{\varepsilon T \operatorname{meas}(\Omega)\left(H\left(\rho^{0}\right)+C_{1}\right)}
\end{aligned}
$$

where we used $\tau k \leq T$. We conclude from (47) that

$$
\left|\left\|\rho_{i}^{k}\right\|_{L^{1}(\Omega)}-\left\|\rho_{i}^{0}\right\|_{L^{1}(\Omega)}\right| \leq \sqrt{\varepsilon T \operatorname{meas}(\Omega)\left(H\left(\rho^{0}\right)+C_{1}\right)} .
$$

Given $0<\gamma<1$, let $\varepsilon>0$ satisfy

$$
\sqrt{\varepsilon} \leq \frac{\gamma \min _{1 \leq j \leq N}\left\|\rho_{j}^{0}\right\|_{L^{1}(\Omega)}}{\sqrt{T \operatorname{Tmeas}(\Omega)\left(H\left(\rho^{0}\right)+C_{1}\right)}} .
$$

This proves (45).

For $i=N+1$, we estimate

$$
\begin{aligned}
\left|\left\|\rho_{N+1}^{k}\right\|_{L^{1}(\Omega)}-\left\|\rho_{N+1}^{0}\right\|_{L^{1}(\Omega)}\right| & =\left|\int_{\Omega}\left(1-\sum_{i=1}^{N} \rho_{i}^{k}\right) d z-\int_{\Omega}\left(1-\sum_{i=1}^{N} \rho_{i}^{0}\right) d z\right| \\
& \leq \sum_{i=1}^{N}\left|\left\|\rho_{i}^{k}\right\|_{L^{1}(\Omega)}-\left\|\rho_{i}^{0}\right\|_{L^{1}(\Omega)}\right| \leq \gamma \sum_{i=1}^{N}\left\|\rho_{i}^{0}\right\|_{L^{1}(\Omega)}
\end{aligned}
$$


which proves (46). From this estimate follows that

$$
\left\|\rho_{N+1}^{k}\right\|_{L^{1}(\Omega)} \geq\left\|\rho_{N+1}^{0}\right\|_{L^{1}(\Omega)}-\gamma \sum_{i=1}^{N}\left\|\rho_{i}^{0}\right\|_{L^{1}(\Omega)} .
$$

Hence, defining

$$
\gamma_{0}=\frac{\left\|\rho_{N+1}^{0}\right\|_{L^{1}(\Omega)}}{2 \sum_{i=1}^{N}\left\|\rho_{i}^{0}\right\|_{L^{1}(\Omega)}}
$$

and choosing $0<\gamma<\min \left\{1, \gamma_{0}\right\}$, we deduce that $\left\|\rho_{N+1}^{k}\right\|_{L^{1}(\Omega)} \geq \frac{1}{2}\left\|\rho_{N+1}^{0}\right\|_{L^{1}(\Omega)}$.

Lemma 16 (Uniform $L^{1}$ norms for $c^{k}$ ). With $\gamma$ as in Lemma 15, it holds that

$$
\left|\left\|c^{k}\right\|_{L^{1}(\Omega)}-\left\|c^{0}\right\|_{L^{1}(\Omega)}\right| \leq M_{0} \gamma\left\|c^{0}\right\|_{L^{1}(\Omega)},
$$

where $M_{0}=\max _{1 \leq i \leq N}\left|1-M_{i} / M_{N+1}\right|$.

Proof. We employ the definitions $c^{k}=\sum_{i=1}^{N+1} \rho_{i}^{k} / M_{i}$ and $\sum_{i=1}^{N+1} \rho_{i}^{k}=1$ and the estimate (45) to obtain

$$
\begin{aligned}
& \left|\left\|c^{k}\right\|_{L^{1}(\Omega)}-\left\|c^{0}\right\|_{L^{1}(\Omega)}\right|=\left|\sum_{i=1}^{N+1} \frac{1}{M_{i}} \int_{\Omega}\left(\rho_{i}^{k}-\rho_{i}^{0}\right) d z\right| \\
& \quad=\left|\sum_{i=1}^{N}\left(\frac{1}{M_{i}}-\frac{1}{M_{N+1}}\right) \int_{\Omega}\left(\rho_{i}^{k}-\rho_{i}^{0}\right) d z\right| \leq M_{0} \sum_{i=1}^{N} \frac{1}{M_{i}}\left|\left\|\rho_{i}^{k}\right\|_{L^{1}(\Omega)}-\left\|\rho_{i}^{0}\right\|_{L^{1}(\Omega)}\right| \\
& \quad \leq M_{0} \gamma \sum_{i=1}^{N} \frac{\left\|\rho_{i}^{0}\right\|_{L^{1}(\Omega)}}{M_{i}} \leq M_{0} \gamma\left\|c^{0}\right\|_{L^{1}(\Omega)} .
\end{aligned}
$$

which finishes the proof.

Now, we turn to the proof of Theorem 2 which is divided into several steps.

Step 1: Relative entropy dissipation inequality. Let $\left(u^{k}, w^{k}\right) \in \mathcal{V} \times \widetilde{H}^{2}\left(\Omega ; \mathbb{R}^{N}\right)$ be a solution to (29) and (31) which exists according to Lemma 12. We introduce the following notation:

$$
\begin{aligned}
& \rho^{k}=\left(\rho_{1}^{k}, \ldots, \rho_{N+1}^{k}\right)=\left(\rho_{1}\left(w^{k}\right), \ldots, \rho_{N+1}\left(w^{k}\right)\right), \quad w^{k}=\left(w_{1}^{k}, \ldots, w_{N}^{k}\right), \\
& \bar{\rho}^{k}=\left(\bar{\rho}_{1}^{k}, \ldots, \bar{\rho}_{N+1}^{k}\right), \quad \bar{x}^{k}=\left(\bar{x}_{1}^{k}, \ldots, \bar{x}_{N+1}^{k}\right), \quad \bar{w}^{k}=\left(\bar{w}_{1}^{k}, \ldots, \bar{w}_{N}^{k}\right),
\end{aligned}
$$

where $\bar{\rho}_{i}^{k}=\operatorname{meas}(\Omega)^{-1}\left\|\rho_{i}^{k}\right\|_{L^{1}(\Omega)}, \bar{c}^{k}=\operatorname{meas}(\Omega)^{-1}\left\|c^{k}\right\|_{L^{1}(\Omega)}, \quad \bar{x}_{i}^{k}=\bar{\rho}_{i}^{k} /\left(\bar{c}^{k} M_{i}\right)$ for $i=$ $1, \ldots, N+1$, and $\bar{w}_{i}^{k}=\ln \left(\bar{x}_{i}^{k}\right) / M_{i}-\ln \left(\bar{x}_{N+1}^{k}\right) / M_{N+1}$ for $i=1, \ldots, N$. It holds that

$$
\bar{c}^{k}=\sum_{i=1}^{N+1} \frac{\bar{\rho}_{i}^{k}}{M_{i}}, \quad \sum_{i=1}^{N+1} \bar{\rho}_{i}^{k}=\sum_{i=1}^{N+1} \bar{x}_{i}^{k}=1 .
$$

With the test function $w^{k}-\bar{w}^{k}$ in (31) we obtain

$$
\frac{1}{\tau} \int_{\Omega}\left(\rho^{\prime}\left(w^{k}\right)-\rho^{\prime}\left(w^{k-1}\right)\right) \cdot\left(w^{k}-\bar{w}^{k}\right) d z+\int_{\Omega} \nabla w^{k}: B\left(w^{k}\right) \nabla w^{k} d z
$$




$$
+\int_{\Omega}\left(\left(u^{k} \cdot \nabla\right) \rho^{\prime}\left(w^{k}\right)\right) \cdot\left(w^{k}-\bar{w}^{k}\right) d z+\varepsilon \int_{\Omega}\left(\left|\Delta w^{k}\right|^{2}+w^{k} \cdot\left(w^{k}-\bar{w}^{k}\right)\right) d z=0 .
$$

If $k=1$, we write $\left(\rho_{1}, \ldots, \rho_{N}\right)$ instead of $\rho^{\prime}\left(w^{k-1}\right)$ in the first integral. The second integral can be estimated according to Lemma 11 and the third integral vanishes in view of Lemma 10. Furthermore, using $w^{k} \cdot\left(w^{k}-\bar{w}^{k}\right) \geq \frac{1}{2}\left(\left|w^{k}\right|^{2}-\left|\bar{w}^{k}\right|\right)^{2}$, the fourth integral can be written as

$$
\int_{\Omega}\left(\left|\Delta w^{k}\right|^{2}+w^{k} \cdot\left(w^{k}-\bar{w}^{k}\right)\right) d z \geq \frac{1}{2} \int_{\Omega}\left(\left|w^{k}\right|^{2}-\left|\bar{w}^{k}\right|^{2}\right) d z \geq-\frac{1}{2} \int_{\Omega}\left|\bar{w}^{k}\right|^{2} d z .
$$

It remains to treat the first integral in (51). For this, we employ the formulation (19) of $w^{k}$ and $\rho_{N+1}^{k}=1-\sum_{i=1}^{N} \rho_{i}^{k}$ :

$$
\begin{aligned}
\left(\rho^{\prime}\left(w^{k}\right)-\rho^{\prime}\left(w^{k-1}\right)\right) \cdot w^{k} & =\sum_{i=1}^{N}\left(\rho_{i}^{k}-\rho_{i}^{k-1}\right)\left(\frac{\ln x_{i}^{k}}{M_{i}}-\frac{\ln x_{N+1}^{k}}{M_{N+1}}\right)=\sum_{i=1}^{N+1}\left(\rho_{i}^{k}-\rho_{i}^{k-1}\right) \frac{\ln x_{i}^{k}}{M_{i}} \\
& =\sum_{i=1}^{N+1}\left(c^{k} x_{i}^{k}-c^{k-1} x_{i}^{k-1}\right) \ln x_{i}^{k}=\left(c^{k} x^{k}-c^{k-1} x^{k-1}\right) \cdot \ln x^{k} .
\end{aligned}
$$

Similarly, $\left(\rho^{\prime}\left(w^{k}\right)-\rho^{\prime}\left(w^{k-1}\right)\right) \cdot \bar{w}^{k}=\left(c^{k} x^{k}-c^{k-1} x^{k-1}\right) \cdot \ln \bar{x}^{k}$. Therefore, the first integral becomes

$$
\begin{aligned}
& \int_{\Omega}\left(\rho^{\prime}\left(w^{k}\right)-\rho^{\prime}\left(w^{k-1}\right)\right) \cdot\left(w^{k}-\bar{w}^{k}\right) d z=\int_{\Omega}\left(c^{k} x^{k}-c^{k-1} x^{k-1}\right) \cdot \ln \frac{x^{k}}{\bar{x}^{k}} d z \\
& \quad=\int_{\Omega}\left(c^{k} x^{k}-c^{k-1} x^{k-1}\right) \cdot \ln \frac{x^{k}}{\bar{x}^{0}} d z+\int_{\Omega}\left(c^{k} x^{k}-c^{k-1} x^{k-1}\right) \cdot \ln \frac{\bar{x}^{0}}{\bar{x}^{k}} d z=I_{1}+I_{2} .
\end{aligned}
$$

First, we estimate $I_{1}$. To this end, we use the convexity of $h\left(\rho^{\prime}\right)$ :

$$
h\left(\rho^{\prime}\left(w^{k}\right)\right)-h\left(\rho^{\prime}\left(w^{k-1}\right)\right) \leq w^{k} \cdot\left(\rho^{\prime}\left(w^{k}\right)-\rho^{\prime}\left(w^{k-1}\right)\right)=\left(c^{k} x^{k}-c^{k-1} x^{k-1}\right) \cdot \ln x^{k} .
$$

Then definitions (12) of the relative entropy $H^{*}$ and (7) of the entropy density $h\left(\rho^{\prime}\right)$ give

$$
\begin{aligned}
H^{*}\left(\rho^{k}\right)-H^{*}\left(\rho^{k-1}\right)= & \sum_{i=1}^{N+1} \int_{\Omega}\left(c^{k} x_{i}^{k} \ln x_{i}^{k}-c^{k-1} x_{i}^{k-1} \ln x_{i}^{k-1}\right) d z \\
& -\int_{\Omega}\left(c^{k} x^{k}-c^{k-1} x^{k-1}\right) \cdot \ln \bar{x}^{0} d z \\
= & \int_{\Omega}\left(h\left(\rho^{\prime}\left(w^{k}\right)\right)-h\left(\rho^{\prime}\left(w^{k-1}\right)\right)\right) d z+\sum_{i=1}^{N+1} \int_{\Omega}\left(c^{k} x_{i}^{k}-c^{k-1} x_{i}^{k-1}\right) d z \\
& -\int_{\Omega}\left(c^{k}-c^{k-1}\right) d z-\int_{\Omega}\left(c^{k} x^{k}-c^{k-1} x^{k-1}\right) \cdot \ln \bar{x}^{0} d z .
\end{aligned}
$$

Since $\sum_{i=1}^{N+1} \int_{\Omega} c^{k} x_{i}^{k} d z=\int_{\Omega} c^{k} d z$, the second and third integrals on the right-hand side cancel. We employ (52) to find that

$$
H^{*}\left(\rho^{k}\right)-H^{*}\left(\rho^{k-1}\right) \leq \int_{\Omega}\left(c^{k} x^{k}-c^{k-1} x^{k-1}\right) \cdot \ln x^{k} d z-\int_{\Omega}\left(c^{k} x^{k}-c^{k-1} x^{k-1}\right) \cdot \ln \bar{x}_{i}^{0} d z=I_{1} .
$$


Next, we estimate $I_{2}$. Let $0<\gamma<\min \left\{\frac{1}{2}, \gamma_{0},\left(2 M_{0}\right)^{-1}\right\}$, where $M_{0}$ is defined in Lemma 16. We infer from Lemmas 15 and 16 and from the definition (50) of $\gamma_{0}$ the following bounds:

$$
\begin{aligned}
& \frac{1-M_{0} \gamma}{1+\gamma} \leq \frac{\bar{x}_{i}^{0}}{\bar{x}_{i}^{k}}=\frac{\left\|\rho_{i}^{0}\right\|_{L^{1}(\Omega)}\left\|c^{k}\right\|_{L^{1}(\Omega)}}{\left\|\rho_{i}^{k}\right\|_{L^{1}(\Omega)}\left\|c^{0}\right\|_{L^{1}(\Omega)}} \leq \frac{1+M_{0} \gamma}{1-\gamma}, \quad i=1, \ldots, N, \\
& \frac{1-M_{0} \gamma}{1+\gamma /\left(2 \gamma_{0}\right)} \leq \frac{\bar{x}_{N+1}^{0}}{\bar{x}_{N+1}^{k}}=\frac{\left\|\rho_{N+1}^{0}\right\|_{L^{1}(\Omega)}\left\|c^{k}\right\|_{L^{1}(\Omega)}}{\left\|\rho_{N+1}^{k}\right\|_{L^{1}(\Omega)}\left\|c^{0}\right\|_{L^{1}(\Omega)}} \leq \frac{1+M_{0} \gamma}{1-\gamma /\left(2 \gamma_{0}\right)} .
\end{aligned}
$$

Thus, taking into account $\sum_{i=1}^{N+1} x_{i}^{k}=1$, we obtain

$$
\begin{aligned}
I_{2} \geq & \sum_{i=1}^{N} \int_{\Omega} c^{k} x_{i}^{k} d z \ln \frac{1-M_{0} \gamma}{1+\gamma}+\int_{\Omega} c^{k} x_{N+1}^{k} d z \ln \frac{1-M_{0} \gamma}{1+\gamma /\left(2 \gamma_{0}\right)} \\
& -\sum_{i=1}^{N} \int_{\Omega} c^{k-1} x_{i}^{k-1} d z \ln \frac{1+M_{0} \gamma}{1-\gamma}-\int_{\Omega} c^{k-1} x_{N+1}^{k-1} d z \ln \frac{1+M_{0} \gamma}{1-\gamma /\left(2 \gamma_{0}\right)} \\
\geq & \int_{\Omega} c^{k} d z \ln \frac{1-M_{0} \gamma}{(1+\gamma)\left(1+\gamma /\left(2 \gamma_{0}\right)\right)}-\int_{\Omega} c^{k-1} d z \ln \frac{1+M_{0} \gamma}{(1-\gamma)\left(1-\gamma /\left(2 \gamma_{0}\right)\right)} .
\end{aligned}
$$

Because of $c^{k} \leq\left(\min _{1 \leq i \leq N+1} M_{i}\right)^{-1}=M_{*}^{-1}$ (see (17) $)$, we conclude that

$$
I_{2} \geq-C_{2}(\gamma):=-\operatorname{meas}(\Omega) M_{*}^{-1} \ln \frac{\left(1+M_{0} \gamma\right)(1+\gamma)\left(1+\gamma /\left(2 \gamma_{0}\right)\right)}{\left(1-M_{0} \gamma\right)(1-\gamma)\left(1-\gamma /\left(2 \gamma_{0}\right)\right)}
$$

Therefore, the first integral in (51) is bounded as follows:

$$
\int_{\Omega}\left(\rho^{\prime}\left(w^{k}\right)-\rho^{\prime}\left(w^{k-1}\right)\right) \cdot\left(w^{k}-\bar{w}^{k}\right) d z \geq H^{*}\left(\rho^{k}\right)-H^{*}\left(\rho^{k-1}\right)-C_{2}(\gamma) .
$$

Summarizing, (51) can be estimated as

$$
H^{*}\left(\rho^{k}\right)-H^{*}\left(\rho^{k-1}\right)+C_{B} \tau \int_{\Omega}\left\|\nabla \sqrt{x^{k}}\right\|^{2} d z \leq \frac{\varepsilon \tau}{2} \int_{\Omega}\left|\bar{w}^{k}\right|^{2} d z+C_{2}(\gamma) .
$$

Step 2: Estimate of the relative entropy. We split the relative entropy into two integrals:

$$
H^{*}\left(\rho^{k}\right)=\sum_{i=1}^{N+1} \int_{\Omega} c^{k} x_{i}^{k} \ln \frac{x_{i}^{k}}{\bar{x}_{i}^{k}} d z+\sum_{i=1}^{N+1} \int_{\Omega} c^{k} x_{i}^{k} \ln \frac{\bar{x}_{i}^{k}}{\bar{x}_{i}^{0}} d z=J_{1}+J_{2} .
$$

It follows from (53) and (54) that

$$
\begin{aligned}
J_{2} & \leq \sum_{i=1}^{N} \int_{\Omega} c^{k} x_{i}^{k} d z \ln \frac{1+\gamma}{1-M_{0} \gamma}+\int_{\Omega} c^{k} x_{N+1}^{k} d z \ln \frac{1+\gamma /\left(2 \gamma_{0}\right)}{1-M_{0} \gamma} \\
& \leq C_{3}(\gamma):=\operatorname{meas}(\Omega) M_{*}^{-1} \ln \frac{(1+\gamma)\left(1+\gamma /\left(2 \gamma_{0}\right)\right.}{1-M_{0} \gamma} .
\end{aligned}
$$


The integral $J_{1}$ is also split into two parts:

$$
J_{1}=\sum_{i=1}^{N+1} \int_{\Omega} c^{k} x_{i}^{k} \ln \frac{c^{k} x_{i}^{k} \operatorname{meas}(\Omega)}{\left\|c^{k} x_{i}^{k}\right\|_{L^{1}(\Omega)}} d z+\sum_{i=1}^{N+1} \int_{\Omega} c^{k} x_{i}^{k} \ln \frac{\left\|c^{k} x_{i}^{k}\right\|_{L^{1}(\Omega)}}{c^{k} \bar{x}_{i}^{k} \operatorname{meas}(\Omega)} d z=J_{11}+J_{12} .
$$

Inserting the definitions $x_{i}^{k}=\rho_{i}^{k} /\left(c^{k} M_{i}\right)$ and $\bar{x}_{i}^{k}=\bar{\rho}_{i}^{k} /\left(\bar{c}^{k} M_{i}\right)$ and using Jensen's inequality for the convex function $s \mapsto s \ln s(s>0)$, we obtain

$$
J_{12}=\sum_{i=1}^{N+1} \int_{\Omega} c^{k} x_{i}^{k} \ln \frac{\bar{c}^{k}}{c^{k}} d z=\int_{\Omega} c^{k} \ln \frac{\bar{c}^{k}}{c^{k}} d z=\left\|c^{k}\right\|_{L^{1}(\Omega)} \ln \bar{c}^{k}-\left\|c^{k} \ln c^{k}\right\|_{L^{1}(\Omega)} \leq 0 .
$$

The estimate of $J_{11}$ is more involved. We employ the logarithmic Sobolev inequality

$$
\int_{\Omega} u^{2} \ln \frac{u^{2}}{\bar{u}^{2}} d z \leq C_{L} \int_{\Omega}|\nabla u|^{2} d z, \quad \bar{u}^{2}=\frac{1}{\operatorname{meas}(\Omega)} \int_{\Omega} u^{2} d z,
$$

where $u \in H^{1}(\Omega)$, and $C_{L}>0$ depends only on $\Omega$ [13]. Then

$$
J_{11} \leq C_{L} \sum_{i=1}^{N+1} \int_{\Omega}\left|\nabla \sqrt{c^{k} x_{i}^{k}}\right|^{2} d z
$$

Since

$$
\sum_{i=1}^{N+1}\left|\nabla \sqrt{c^{k} x_{i}^{k}}\right|^{2} \leq 2 \sum_{i=1}^{N+1} x_{i}^{k}\left|\nabla \sqrt{c^{k}}\right|^{2}+2 \sum_{i=1}^{N+1} c^{k}\left|\nabla \sqrt{x_{i}^{k}}\right|^{2}=2\left|\nabla \sqrt{c^{k}}\right|^{2}+2 c^{k}\left\|\nabla \sqrt{x^{k}}\right\|^{2},
$$

we obtain

$$
J_{11} \leq 2 C_{L} \int_{\Omega}\left|\nabla \sqrt{c^{k}}\right|^{2} d z+2 C_{L} M_{*}^{-1} \int_{\Omega}\left\|\nabla \sqrt{x^{k}}\right\|^{2} d z .
$$

We claim that the first integral can be estimated by a multiple of the second one. Indeed, by the Cauchy-Schwarz inequality, the definition of $c^{k}$ according to Lemma 6, and the bound (17), it follows that

$$
\begin{aligned}
\left|\nabla \sqrt{c^{k}}\right|^{2} & =\frac{1}{4 c^{k}}\left|\frac{-\sum_{i=1}^{N+1} M_{i} \nabla x_{i}^{k}}{\left(\sum_{i=1}^{N+1} M_{i} x_{i}^{k}\right)^{2}}\right|^{2}=\left(c^{k}\right)^{3}\left|\sum_{i=1}^{N+1} M_{i} \sqrt{x_{i}^{k}} \nabla \sqrt{x_{i}^{k}}\right|^{2} \\
& \leq\left(c^{k}\right)^{3} \sum_{i=1}^{N+1} M_{i}^{2} x_{i}^{k} \sum_{i=1}^{N+1}\left|\nabla \sqrt{x_{i}^{k}}\right|^{2} \leq M_{*}^{-3} M^{* 2}\left\|\nabla \sqrt{x^{k}}\right\|^{2},
\end{aligned}
$$

recalling that $M_{*}=\min _{1 \leq i \leq N+1} M_{i}$ and setting $M^{*}=\max _{1 \leq i \leq N+1} M_{i}$. Thus, we can estimate $J_{11}$ as follows:

$$
J_{11} \leq 2 C_{L} M_{*}^{-1}\left(M_{*}^{-2} M^{* 2}+1\right) \int_{\Omega}\left\|\nabla \sqrt{x^{k}}\right\|^{2} d z .
$$

Combining the above estimates, we conclude that

$$
H^{*}\left(\rho^{k}\right) \leq C_{3}(\gamma)+2 C_{L} M_{*}^{-1}\left(M_{*}^{-2} M^{* 2}+1\right) \int_{\Omega}\left\|\nabla \sqrt{x^{k}}\right\|^{2} d z .
$$


Step 3: End of the proof. Replacing the entropy dissipation term involving $\sqrt{x^{k}}$ in (56) by the above estimate for $H^{*}\left(\rho^{k}\right)$, we find that

$$
\left(1+C_{4} \tau\right) H^{*}\left(\rho^{k}\right) \leq H^{*}\left(\rho^{k-1}\right)+\frac{\varepsilon \tau}{2} \int_{\Omega}\left|\bar{w}^{k}\right|^{2} d z+C_{\gamma},
$$

where $C_{4}=\frac{1}{2} C_{B} C_{L}^{-1} M_{*}\left(M_{*}^{-2} M^{* 2}+1\right)^{-1}$ and $C_{\gamma}=C_{2}(\gamma)+\frac{1}{2} C_{3}(\gamma) C_{L}^{-1} M_{*}\left(M_{*}^{-2} M^{* 2}+1\right)^{-1}$. Note that according to definitions (55) and (57), we have $C_{\gamma} \rightarrow 0$ as $\gamma \rightarrow 0$.

We need to estimate the integral involving $w^{k}$. For this, we observe that (53)-(54) and the upper bound for $\gamma$ imply that $\frac{1}{3} \leq \bar{x}_{i}^{0} / \bar{x}_{i}^{k} \leq 3$ for $i=1, \ldots, N+1$. This provides some uniform bounds for $\bar{x}_{i}^{k}$,

$$
0<\frac{M_{*} \min _{1 \leq i \leq N+1}\left\|\rho_{i}^{0}\right\|_{L^{1}(\Omega)}}{3 M^{*} \sum_{i=1}^{N+1}\left\|\rho_{i}^{0}\right\|_{L^{1}(\Omega)}} \leq \frac{\bar{x}_{i}^{0}}{3} \leq \bar{x}_{i}^{k} \leq 3 \bar{x}_{i}^{0} \leq 3, \quad i=1, \ldots, N+1,
$$

which allow us to estimate $w^{k}$ :

$$
\int_{\Omega}\left|\bar{w}^{k}\right|^{2} d z \leq \sum_{i=1}^{N} \int_{\Omega}\left(\left|\frac{\ln \bar{x}_{i}^{k}}{M_{i}}\right|+\left|\frac{\ln \bar{x}_{N+1}^{k}}{M_{N+1}}\right|\right)^{2} d z \leq C_{5}
$$

where $C_{5}>0$ depends on $\Omega, \rho^{0}, M_{*}$, and $M^{*}$. Hence, (158) becomes

$$
H^{*}\left(\rho^{k}\right) \leq\left(1+C_{4} \tau\right)^{-1} H^{*}\left(\rho^{k-1}\right)+\left(\frac{\varepsilon \tau}{2} C_{5}+C_{\gamma}\right)\left(1+C_{4} \tau\right)^{-1} .
$$

Solving this recursion, we infer that

$$
H^{*}\left(\rho^{k}\right) \leq\left(1+C_{4} \tau\right)^{-1} H^{*}\left(\rho^{0}\right)+\left(\frac{\varepsilon \tau}{2} C_{5}+C_{\gamma}\right) \sum_{i=1}^{k}\left(1+C_{4} \tau\right)^{-i} .
$$

Using $\sum_{i=1}^{k}\left(1+C_{4} \tau\right)^{-i} \leq 1 /\left(C_{4} \tau\right)$, it follows that

$$
H^{*}\left(\rho^{(\tau)}(\cdot, t)\right) \leq\left(1+C_{4} \tau\right)^{-t / \tau} H^{*}\left(\rho^{0}\right)+\frac{\varepsilon C_{5}}{2 C_{4}}+\frac{C_{\gamma}}{C_{4} \tau}, \quad 0<t<T .
$$

Now, we take $\tau=\tau(\gamma)=\sqrt{C_{\gamma}}$ and $\varepsilon=\varepsilon(\gamma)$ according to (49). In the limit $\gamma \rightarrow 0$, it follows that $C_{\gamma} / \tau(\gamma) \rightarrow 0, \varepsilon(\gamma) \rightarrow 0$, and $\tau(\gamma) \rightarrow 0$ so that $\rho_{i}^{(\tau)} \rightarrow \rho_{i}$ strongly in $L^{2}\left(0, T ; L^{2}(\Omega)\right)$ for $i=1, \ldots, N+1$. This gives in the limit $\gamma \rightarrow 0$

$$
H^{*}(\rho(\cdot, t)) \leq e^{-C_{4} t} H^{*}\left(\rho^{0}\right), \quad t \geq 0,
$$

and, taking into account Lemmas 15] and 16, we conclude the $L^{1}$ conservation for $\rho_{i}$ and $c$ :

$$
\int_{\Omega} \rho_{i} d z=\int_{\Omega} \rho_{i}^{0} d z, \quad \int_{\Omega} c d z=\int_{\Omega} c^{0} d z
$$

where $c^{0}=\sum_{j=1}^{N+1} \rho_{j}^{0} / M_{j}$ and $i=1, \ldots, N+1$.

It remains to estimate $x_{i}-\bar{x}_{i}^{0}$ in the $L^{1}$ norm. Defining

$$
f_{i}=\frac{c x_{i}}{\int_{\Omega} c^{0} x_{i}^{0} d z}, \quad g_{i}=\frac{c}{\int_{\Omega} c^{0} d z}
$$


the entropy $H^{*}(\rho)=\sum_{i=1}^{N+1} \int_{\Omega} c x_{i} \ln \left(x_{i} / \bar{x}_{i}^{0}\right) d z$ can be written as

$$
H^{*}(\rho)=\sum_{i=1}^{N+1} \int_{\Omega} c^{0} x_{i}^{0} d z \int_{\Omega} f_{i} \ln \frac{f_{i}}{g_{i}} d z
$$

where we employed the identity

$$
\frac{f_{i}}{g_{i}}=\frac{x_{i} \int_{\Omega} c^{0} d z}{\int_{\Omega} c^{0} x_{i}^{0} d z}=\frac{M_{i} x_{i} \int_{\Omega} c^{0} d z}{\int_{\Omega} \rho_{i}^{0} d z}=\frac{M_{i} x_{i} \bar{c}^{0}}{\bar{\rho}_{i}^{0}}=\frac{x_{i}}{\bar{x}_{i}^{0}} .
$$

Finally, using

$$
c \bar{x}_{i}^{0}=\frac{c \bar{\rho}_{i}^{0}}{\bar{c}^{0} M_{i}}=\frac{c \int_{\Omega} \rho_{i}^{0} d z}{\int_{\Omega} c^{0} d z M_{i}}=\frac{c \int_{\Omega} c^{0} x_{i}^{0} d z}{\int_{\Omega} c^{0} d z}=\int_{\Omega} c^{0} x_{i}^{0} d z g_{i}
$$

and the Csiszár-Kullback inequality with constant $C_{K}>0$ (see, e.g., [13, 19]), we find that

$$
\begin{aligned}
\left\|c x_{i}-c \bar{x}_{i}^{0}\right\|_{L^{1}(\Omega)}^{2} & =\left(\int_{\Omega} c^{0} x_{i}^{0} d z\right)^{2}\left\|f_{i}-g_{i}\right\|_{L^{1}(\Omega)}^{2} \leq \int_{\Omega} c^{0} x_{i}^{0} d z\left(\int_{\Omega} \frac{\rho_{i}^{0}}{M_{i}} d z\right) C_{K} \int_{\Omega} f_{i} \ln \frac{f_{i}}{g_{i}} d z \\
& \leq M_{i}^{-1} C_{K}\left\|\rho_{i}^{0}\right\|_{L^{1}(\Omega)} H^{*}(\rho) .
\end{aligned}
$$

Together with (59), the conclusion of the theorem follows.

\section{REFERENCES}

[1] N. Amundson, E. Morano, and R. Sanders. Techniques for the numerical solution of steady reactiondiffusion systems employing Stefan-Maxwell diffusion. East-West J. Numer. Math. 6 (1998), 9-25.

[2] N. Amundson, T.-W. Pan, and V. Paulsen. Diffusing with Stefan and Maxwell. AIChE Journal 49 (2003), 813-830.

[3] P. Andries, K. Aoki, and B. Perthame. A consistent BGK-type model for gas mixtures. J. Statist. Phys. 106 (2002), 993-1018.

[4] D. Bothe. On the Maxwell-Stefan equations to multicomponent diffusion. In: Progress in Nonlinear Differential Equations and their Applications, pp. 81-93. Springer, Basel, 2011.

[5] D. Bothe, A. Fischer, and J. Saal. Global well-posedness and stability of electro-kinetic flows. Preprint, 2013. arXiv: 1206.1457.

[6] L. Boudin, B. Grec, and F. Salvarani. A mathematical and numerical analysis of the Maxwell-Stefan diffusion equations. Discr. Contin. Dyn. Sys. B 5 (2012), 1427-1440.

[7] L. Chen and A. Jüngel. Analysis of a multi-dimensional parabolic population model with strong cross-diffusion. SIAM J. Math. Anal. 36 (2004), 301-322.

[8] M. Dreher and A. Jüngel. Compact families of piecewise constant functions in $L^{p}(0, T ; B)$. Nonlin. Anal. 75 (2012), 3072-3077.

[9] V. Giovangigli. Convergent iterative methods for multicomponent diffusion. IMPACT Comput. Sci. Engin. 3 (1991), 244-276.

[10] V. Giovangigli. Multicomponent Flow Modeling. Birkhäuser, Basel, 1999.

[11] V. Giovangigli. Plane flames with multicomponent transport and complex chemistry. Math. Models Meth. Appl. Sci. 9 (1999), 337-378.

[12] J. Griepentrog. On the unique solvability of a nonlocal phase separation problem for multicomponent systems. Banach Center Publ. 66 (2004), 153-164.

[13] A. Jüngel. Entropy dissipation methods for nonlinear partial differential equations. Lecture Notes, 2012. http://www. jungel.at.vu. 
[14] A. Jüngel and I. Stelzer. Existence analysis of Maxwell-Stefan systems for multicomponent mixtures. To appear in SIAM J. Math. Anal., 2013. arXiv:1211.2394.

[15] O. Manley, M. Marion, and R. Temam. Fully nonlinear multispecies reaction-diffusion equations. Appl. Math. Lett. 8 (1995), 7-11.

[16] P. Mucha, M. Pokorný, and E. Zatorska. Approximate solutions to a model of two-component reactive flow. Preprint no. 2012-030, University of Warsaw2012. http://mmns.mimuw.edu.pl.

[17] P. Mucha, M. Pokorný, and E. Zatorska. Chemically reacting mixtures in terms of degenerated parabolic setting. J. Math. Phys. 54 (2013), 071501, 17 pages.

[18] R. Temam. Navier-Stokes Equations. Theory and Numerical Analysis. Third edition. North-Holland, Amsterdam, 1984.

[19] A. Unterreiter, A. Arnold, P. Markowich, and G. Toscani. On generalized Csiszár-Kullback inequalities. Monatsh. Math. 131 (2000), 235-253.

[20] J. Wesselingh and R. Krishna. Mass Transfer in Multicomponent Mixtures. Delft University Press, Delft, 2000.

[21] E. Zatorska. On the steady flow of a multicomponent, compressible, chemically reacting gas. Nonlinearity 24 (2011), 3267-3278.

[22] E. Zatorska. On the flow of chemically reacting gaseous mixture. J. Diff. Eqs. 253 (2012), 3471-3500.

[23] E. Zeidler. Nonlinear Functional Analysis and its Applications, Vol. II/A. Springer, New York, 1990.

School of Sciences, Beijing University of Posts and Telecommunications, Beijing 100876 , CHINA

E-mail address: buptxchen@yahoo.com

Institute for Analysis and Scientific Computing, Vienna University of Technology, Wiedner Hauptstrasse 8-10, 1040 Wien, Austria

E-mail address: juengel@tuwien.ac.at 